TRANSACTIONS OF THE

AMERICAN MATHEMATICAL SOCIETY

Volume 359, Number 12, December 2007, Pages 6079-6110

S 0002-9947(07)04346-2

Article electronically published on May 7, 2007

\title{
QUADRATIC MAPS AND BOCKSTEIN CLOSED GROUP EXTENSIONS
}

\author{
JONATHAN PAKIANATHAN AND ERGÜN YALÇIN
}

\begin{abstract}
Let $E$ be a central extension of the form $0 \rightarrow V \rightarrow G \rightarrow W \rightarrow 0$ where $V$ and $W$ are elementary abelian 2-groups. Associated to $E$ there is a quadratic map $Q: W \rightarrow V$, given by the 2-power map, which uniquely determines the extension. This quadratic map also determines the extension class $q$ of the extension in $H^{2}(W, V)$ and an ideal $I(q)$ in $H^{2}(G, \mathbb{Z} / 2)$ which is generated by the components of $q$. We say that $E$ is Bockstein closed if $I(q)$ is an ideal closed under the Bockstein operator.

We find a direct condition on the quadratic map $Q$ that characterizes when the extension is Bockstein closed. Using this characterization, we show for example that quadratic maps induced from the fundamental quadratic map $Q_{\mathfrak{g l}_{n}}: \mathfrak{g l}_{n}\left(\mathbb{F}_{2}\right) \rightarrow \mathfrak{g l}_{n}\left(\mathbb{F}_{2}\right)$ given by $Q(\mathbb{A})=\mathbb{A}+\mathbb{A}^{2}$ yield Bockstein closed extensions.

On the other hand, it is well known that an extension is Bockstein closed if and only if it lifts to an extension $0 \rightarrow M \rightarrow \widetilde{G} \rightarrow W \rightarrow 0$ for some $\mathbb{Z} / 4[W]$ lattice $M$. In this situation, one may write $\beta(q)=L q$ for a "binding matrix" $L$ with entries in $H^{1}(W, \mathbb{Z} / 2)$. We find a direct way to calculate the module structure of $M$ in terms of $L$. Using this, we study extensions where the lattice $M$ is diagonalizable/triangulable and find interesting equivalent conditions to these properties.
\end{abstract}

\section{INTRODUCTION}

It is of course well known that in studying the cohomology of finite groups the cohomology of $p$-groups plays a fundamental part. Any $p$-group $P$ has a central series with elementary abelian factors (for example the central Frattini series) and so, in principle, its cohomology can be studied by iteratively considering central extensions with elementary abelian kernel.

The simplest nontrivial situation is given by $p$-groups $P$ that fit in a central short exact sequence

$$
0 \rightarrow V \rightarrow P \rightarrow W \rightarrow 0
$$

where $V$ and $W$ are elementary abelian $p$-groups (finite $\mathbb{F}_{p}$-vector spaces).

For $p$ odd, such extensions are in bijective correspondence with a choice of $p$ power map $\phi: W \rightarrow V$ which is linear and an alternating bilinear commutator

Received by the editors December 2, 2005.

2000 Mathematics Subject Classification. Primary 20J05; Secondary 17B50, 15A63.

Key words and phrases. Group extensions, quadratic maps, group cohomology, restricted Lie algebras.

The second author was partially supported by a grant from the Turkish Academy of Sciences (TÜBA-GEBİP/2005-16). 
map

$$
[\cdot, \cdot]: W \wedge W \rightarrow V
$$

For $p=2$, such extensions are in bijective correspondence with a quadratic 2-power map $Q: W \rightarrow V$ which has an associated bilinear map given by the commutator. Since the power map is not linear but quadratic in this case, this adds considerable difficulty in this situation.

In this paper we study various algebraic properties of these extensions in the case $p=2$. We start with a review of the above mentioned facts and an explicit description of how to recover the extension class in $H^{2}(W, V)$ of the extension from the quadratic map $Q$. The components of the extension class form a quadratic ideal $I(Q) \subseteq H^{*}\left(W, \mathbb{F}_{2}\right)$.

In order to compute the cohomology of the group $G$ given by the extension, an important condition that is often considered is whether the ideal $I(Q)$ is closed under the Bockstein operator (and hence under the Steenrod algebra). Our first theorem gives a necessary and sufficient condition for the ideal $I(Q)$ to be Bockstein closed based on conditions on the associated quadratic form $Q$ of the extension.

Theorem 1.1. Let $Q: W \rightarrow V$ be a quadratic map, and let $B$ be the bilinear map associated to $Q$. Then, $Q$ is Bockstein closed if and only if there exists a bilinear map $P: V \times W \rightarrow V$ which satisfies the identity

$$
P(Q(x), y)=B(x, y)+P(B(x, y), x)
$$

for all $x, y \in W$.

While this condition might seem mysterious at first, the case of $P=B$ happens when $Q$ is obtained from the 2-power map of a 2-restricted Lie algebra (see Section 9). A fundamental example of a quadratic map is $Q_{\mathfrak{g l}_{n}}: \mathfrak{g l}_{n}\left(\mathbb{F}_{2}\right) \rightarrow \mathfrak{g l}_{n}\left(\mathbb{F}_{2}\right)$ given by

$$
Q_{\mathfrak{g r}_{n}}(\mathbb{A})=\mathbb{A}+\mathbb{A}^{2}
$$

Using the criterion above, it follows that any quadratic map induced from $Q_{\mathfrak{g l}_{n}}$ (by restriction of domain and codomain) has an associated extension which is Bockstein closed.

Let $G$ be the kernel of the mod 2 reduction map $G L_{n}(\mathbb{Z} / 8) \rightarrow G L_{n}(\mathbb{Z} / 2)$. It is easy to see that $G$ fits into a central short exact sequence

$$
0 \rightarrow \mathfrak{g l}_{n}\left(\mathbb{F}_{2}\right) \rightarrow G \rightarrow \mathfrak{g l}_{n}\left(\mathbb{F}_{2}\right) \rightarrow 0
$$

with associated quadratic form $Q_{\mathfrak{g l}_{n}}$. It follows that this extension and its restrictions to suitable subspaces such as $\mathfrak{s l}_{n}\left(\mathbb{F}_{2}\right)$ or $\mathfrak{u}_{n}\left(\mathbb{F}_{2}\right)$ are Bockstein closed. (This is an important ingredient in calculating the cohomology of these groups as rings and as modules over the Steenrod algebra. See for example [1] and [4.)

In the case that $E$ is a Bockstein closed extension, there is a cohomology class $L$ in $H^{1}(W, \operatorname{End}(V))$ such that $\beta(q)=L q$ holds. Here the multiplication $L q$ is given by the composite

$$
H^{1}(W, \operatorname{End}(V)) \otimes H^{2}(W, V) \stackrel{\cup}{\longrightarrow} H^{3}(W, \operatorname{End}(V) \otimes V) \rightarrow H^{3}(W, V),
$$

where the second map is induced by the evaluation map ev $: \operatorname{End}(V) \otimes V \rightarrow V$. In this case we call the element $L$ a binding operator. Note that if we choose a basis for $V$, then we can write $L$ as a matrix with entries in $H^{1}\left(W, \mathbb{F}_{2}\right)$ and express the equation $\beta(q)=L q$ as a matrix equation, where $q$ in the equation denotes the column matrix whose entries are the components of $q$. 
It is well known that the central extension $E: 0 \rightarrow V \rightarrow G \rightarrow W \rightarrow 0$ has a "uniform" lift to an extension $\widetilde{E}: 0 \rightarrow M \rightarrow \widetilde{G} \rightarrow W \rightarrow 0$, where $M$ is a free $\mathbb{Z} / 4$ module with $M / 2 M=V$ and $\widetilde{G} / 2 M=G$, if and only if the extension $E$ is Bockstein closed. (We will reprove this in this paper when proving more general results.) The lifting module $M$ has a $W$-action that is in general nontrivial and this yields a map $\rho_{M}: W \rightarrow G L(M)$, where $G L(M)$ denotes the automorphism group of $M$. Note that $\rho_{M}$ sits in the subgroup $G L(M ; V, V)$ of $G L(M)$ formed by automorphisms of $M$ that induce a trivial action on $M / 2 M$ and $2 M$. In the paper we derive a certain explicit exponent-log correspondence between $\operatorname{Hom}(W, G L(M ; V, V))$ and $H^{1}(W, \operatorname{End}(V))$ and conclude the following:

Theorem 1.2. Let $E: 0 \rightarrow V \rightarrow G \rightarrow W \rightarrow 0$ be a central extension. If $E$ lifts to an extension $\widetilde{E}$ with kernel $M$, then $\log ^{*}\left(\rho_{M}\right) \in H^{1}(W$, End $(V))$ is a binding operator for $E$. Conversely, if $L$ is a binding operator for $E$, then $E$ lifts to an extension $\widetilde{E}$ with kernel $M$ where $M$ has a representation $\exp ^{*}(L) \in \operatorname{Hom}(W, G L(M ; V, V))$.

This allows one to compute a binding operator $L$ from the module structure of a lifting module $M$ and vice-versa. Note that in general the lifting module is not unique, but there is a unique lifting module for every chosen binding operator. In the paper we give examples of extensions which have more than one lifting module and more than one binding operator (see Example 3.5 ).

We then go on in Section 5 to study the conditions for a Bockstein closed extension to have a diagonalizable binding operator $L$ (or equivalently a diagonalizable lifting module $M$ ). We get the following theorem characterizing the diagonalizable situation:

Theorem 1.3. Let $Q: W \rightarrow V$ be a Bockstein closed quadratic map, and let $E: 0 \rightarrow V \rightarrow G \rightarrow W \rightarrow 0$ be the central extension associated to $Q$. Let $q$ denote the extension class for $E$. Then, the following are equivalent:

(i) $Q$ is diagonalizable, i.e., there is a basis for $V$ such that the components of $q$ are individually Bockstein closed.

(ii) There is a choice of basis of $V$ such that the components $q_{1}, \ldots, q_{n}$ of $q$ all decompose as $q_{i}=u_{i} v_{i}$ where $u_{i}, v_{i}$ are linear polynomials.

(iii) There exists a diagonalizable $L \in \operatorname{Hom}(W, \operatorname{End}(V))$ such that $\beta(q)=L q$. This is characterized exactly by the equation $\beta(L)+L^{2}=0$.

(iv) E lifts to an extension with kernel $M$ where $M$ is a direct sum of one dimensional $\mathbb{Z} / 4[W]$-lattices.

(v) E lifts to an extension with kernel $M$ where $M$ is a $\mathbb{Z} / 8[W]$-lattice.

(vi) E lifts to an extension with kernel $M$ where $M$ is a $\mathbb{Z}[W]$-lattice, i.e., E has a uniform integral lifting.

Some of the implications above are well known to experts on this subject. The most interesting implication in the above theorem is that if $E$ has a uniform integral lifting, then the associated quadratic map is diagonalizable (see Proposition 5.6). In other words, the only extensions we get from integral extensions are the diagonalizable ones.

In Section 6, we consider triangulability of extensions and find equivalent conditions to triangulability in terms of the binding operator $L$ and lifting module $M$. We show that if the extension is triangulable, then it has a binding matrix $L$ such that $\beta(L)+L^{2}$ is nilpotent. Using this we give examples of extensions which are not triangulable. 
In Section 7, we show that the components $q_{1}, \ldots, q_{n}$ of $q$ form a regular sequence as long as we make the additional assumptions that $\operatorname{dim}(V)=\operatorname{dim}(W)$ and that $Q$ is effective, i.e., that $Q(w)=0$ is zero only when $w=0$. This turns out to be another important technical tool in calculating the cohomology of the groups given by these extensions.

In Section 8, we consider Bockstein closed 2-power exact extensions (see Definition (7.9). We show that for a Bockstein closed 2-power exact extension the binding operator $L$, and hence lifting module $M$, is unique.

In Section 9, we show that any 2-restricted Lie algebra $\mathfrak{L}$ gives a Bockstein closed extension of the form $E: 0 \rightarrow \mathfrak{L} \rightarrow G \rightarrow \mathfrak{L} \rightarrow 0$ and furthermore that there is a bijection between the 2-restricted Lie algebras over $\mathbb{F}_{2}$ and the Bockstein closed extensions with $P=B$ in Theorem 1.1

In this paper we leave some questions about triangulability of Bockstein closed 2 -power exact extensions unanswered. These questions have equivalent versions in terms of 2-restricted Lie algebras which may be easier to answer than the original versions. The statements of these questions can be found in Sections 8 and 9 ,

\section{Preliminaries And Definitions}

Throughout this section, let $E$ denote a central extension of the form

$$
0 \rightarrow V \stackrel{i}{\longrightarrow} G \stackrel{\pi}{\longrightarrow} W \rightarrow 0
$$

where $V$ and $W$ are elementary abelian 2-groups. In this section, we will explain how the standard theory of group extensions and 2-groups applies to $E$. Then we will make further definitions.

First we will recall how the cohomology class associated to $E$ is defined. A transversal is a function $s: W \rightarrow G$ such that $\pi \circ s=i d$ and $s(0)=1$, where $0 \in W, 1 \in G$ are the respective identities. Given a transversal $s$, we define $f: W \times W \rightarrow V$ by the formula

$$
f(x, y)=i^{-1}\left(s(x+y)^{-1} s(x) s(y)\right) .
$$

We often identify $V$ with its image under $i$ and do not write $i^{-1}$ in our formulas. It is well known that $f$ satisfies the following normalized cocycle identities:

$$
\begin{gathered}
f(x, 0)=f(0, x)=0 \\
f(x+y, z)+f(x, y)=f(y, z)+f(x, y+z) .
\end{gathered}
$$

A function $f: W \times W \rightarrow V$ satisfying these identities is called a (normalized) factor set, and it is known that normalized factor sets correspond to 2-cocycles in the normalized standard cochain complex $C^{*}(W, V)$. When a different transversal is chosen, we get a different factor set, say $f^{\prime}$, such that $f-f^{\prime}$ is a coboundary in $C^{2}(W, V)$. So, for each extension $E$, there is a unique cohomology class $q \in H^{2}(W, V)$, which is called the extension class of $E$. A standard result in group extension theory states that up to a suitable equivalence relation on group extensions the converse also holds (see, for example, Theorem 3.12 in Brown [2]).

Proposition 2.1. There is a one-to-one correspondence between equivalence classes of central extensions of the form $E: 0 \rightarrow V \rightarrow G \rightarrow W \rightarrow 0$ and cohomology classes in $H^{2}(W, V)$, where two extensions $E$ and $E^{\prime}$ are considered equivalent if there is 
a commutative diagram of the following form:

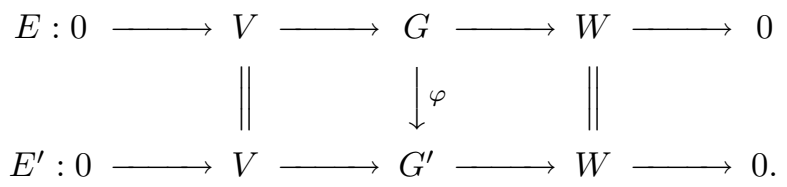

Now, we consider the group theoretical properties of $G$. Let [, ] denote the commutator $[g, h]=g^{-1} h^{-1} g h$, and $g^{h}$ denote the conjugation $h^{-1} g h$. Recall the following identities which are very easy to verify:

$$
\begin{aligned}
{[g, h k] } & =[g, k][g, h]^{k}, \\
{[g h, k] } & =[g, k]^{h}[h, k], \\
(g h)^{2} & =g^{2} h^{2}[h, g]^{h} .
\end{aligned}
$$

Applying these to the extension group $G$, we see that the squaring map induces a map $Q: W \rightarrow V$ defined as $Q(x)=(\hat{x})^{2}$, where $\hat{x}$ denotes an element in $G$ that lifts $x \in W$. Similarly, the commutator induces a symmetric bilinear map $B: W \times W \rightarrow V$ defined as $B(x, y)=[\hat{x}, \hat{y}]$. Note also that since $V$ is a central elementary abelian 2-group, the third row of (1) gives

$$
B(x, y)=Q(x+y)+Q(x)+Q(y)
$$

hence $Q$ is a quadratic map and $B$ is its associated bilinear map.

We will now explain the relation between the quadratic map $Q$ and the extension class $q$. Choosing a basis $\left\{v_{1}, \ldots, v_{n}\right\}$ for $V$, we can write $Q=\left(Q_{1}, \ldots, Q_{n}\right)$ and $B=\left(B_{1}, \ldots, B_{n}\right)$. Also using the isomorphism $H^{2}(W, V) \cong H^{2}(W, \mathbb{Z} / 2) \otimes V$, we can write $q=\left(q_{1}, \ldots, q_{n}\right)$. We have the following:

Proposition 2.2. Let $\left\{w_{1}, \ldots, w_{m}\right\}$ be a basis for $W$, and let $\left\{x_{1}, \ldots, x_{m}\right\}$ denote its dual basis. Under the isomorphism $H^{*}(W, \mathbb{Z} / 2) \cong \mathbb{Z} / 2\left[x_{1}, \ldots, x_{m}\right]$, the cohomology class $q_{k}$ is equal to the quadratic polynomial associated to $Q_{k}$ for each $k$. In other words, as a polynomial in $x_{i}$ 's, the cohomology class $q_{k}$ is of the following form:

$$
q_{k}=\sum_{i} Q_{k}\left(w_{i}\right) x_{i}^{2}+\sum_{i<j} B_{k}\left(w_{i}, w_{j}\right) x_{i} x_{j} .
$$

Proof. Let $E: 0 \rightarrow V \rightarrow G \rightarrow W \rightarrow 0$ be an extension with quadratic map $Q$ and bilinear map $B$. We define $f: W \times W \rightarrow V$ as the bilinear map such that

$$
f\left(w_{i}, w_{j}\right)=\left\{\begin{array}{lll}
B\left(w_{i}, w_{j}\right) & \text { if } & i<j, \\
Q\left(w_{i}\right) & \text { if } & i=j, \\
0 & \text { if } & i>j .
\end{array}\right.
$$

It is clear that, being a bilinear map, $f: W \times W \rightarrow V$ satisfies the factor set conditions. Also note that for every $k$, and for every $w, w^{\prime} \in W$, we have

$$
f_{k}\left(w, w^{\prime}\right)=\sum_{i} Q_{k}\left(w_{i}\right) x_{i}(w) x_{i}\left(w^{\prime}\right)+\sum_{i<j} B_{k}\left(w_{i}, w_{j}\right) x_{i}(w) x_{j}\left(w^{\prime}\right),
$$

so the associated cohomology class for $f_{k}$ is equal to

$$
\sum_{i} Q_{k}\left(w_{i}\right) x_{i}^{2}+\sum_{i<j} B_{k}\left(w_{i}, w_{j}\right) x_{i} x_{j}
$$

under the isomorphism $H^{*}(W, \mathbb{Z} / 2) \cong \mathbb{Z} / 2\left[x_{1}, \ldots, x_{m}\right]$. 
To complete the proof, we just need to show that $f$ is a factor set for $E$. Note that for every $w=a_{1} w_{1}+\cdots+a_{m} w_{m}$ in $W$, we have

$$
f(w, w)=\sum_{i, j} a_{i} a_{j} f\left(w_{i}, w_{j}\right)=\sum_{i} a_{i} Q\left(w_{i}\right)+\sum_{i<j} a_{i} a_{j} B\left(w_{i}, w_{j}\right)=Q(w) .
$$

Hence, the proof follows from the following lemma.

Lemma 2.3. Let $E: 0 \rightarrow V \rightarrow G \rightarrow W \rightarrow 0$ be an extension with quadratic map $Q$. A factor set $f: W \times W \rightarrow V$ is a factor set for $E$ if and only if $f(w, w)=Q(w)$ for all $w \in W$.

Proof. If $f$ is a factor set for $E$ determined by the splitting $s$, then

$$
f(w, w)=s(w+w)^{-1} s(w) s(w)=(s(w))^{2}=Q(w)
$$

holds for all $w \in W$. For the converse, let $f$ be a factor set satisfying $f(w, w)=$ $Q(w)$ for all $w \in W$. Suppose $f^{\prime}$ is a factor set for $E$. Then, $f^{\prime \prime}=f+f^{\prime}$ is a factor set such that $f^{\prime \prime}(w, w)=0$ for all $w \in W$. If $0 \rightarrow V \rightarrow G^{\prime \prime} \stackrel{\pi}{\longrightarrow} W \rightarrow 0$ is the extension with factor set $f^{\prime \prime}$ and transversal $t: W \rightarrow G^{\prime \prime}$, then for every $g \in G^{\prime \prime}$, we have $g^{2}=[t(\pi(g))]^{2}=f^{\prime \prime}(\pi(g), \pi(g))=0$. Thus $G^{\prime \prime}$ is of exponent 2 , and hence an elementary abelian 2-group. This implies that the extension $0 \rightarrow$ $V \rightarrow G^{\prime \prime} \stackrel{\pi}{\longrightarrow} W \rightarrow 0$ splits, and therefore $f^{\prime \prime}$ is cohomologous to zero. This shows that $f^{\prime}$ is also a factor set for $E$.

As a consequence of Propositions 2.1 and 2.2, we obtain the following:

Corollary 2.4. Given a quadratic map $Q: W \rightarrow V$, there is a unique (up to equivalence) central extension

$$
E(Q): 0 \rightarrow V \rightarrow G(Q) \rightarrow W \rightarrow 0
$$

with $Q(w)=(\hat{w})^{2}$ for all $w \in W$.

In fact, there is a natural equivalence between the category of quadratic maps $Q: W \rightarrow V$ and the category of central extensions of the form $E: 0 \rightarrow V \rightarrow G \rightarrow$ $W \rightarrow 0$. We skip the details of this categorical equivalence, since we do not need this for this paper.

We end this section with some examples.

Example 2.5. Let $\mathfrak{g l}_{n}\left(\mathbb{F}_{2}\right)$ denote the vector space of $n \times n$ matrices over $\mathbb{F}_{2}$. Define $Q: \mathfrak{g l}_{n}\left(\mathbb{F}_{2}\right) \rightarrow \mathfrak{g l}_{n}\left(\mathbb{F}_{2}\right)$ by $Q(\mathbb{A})=\mathbb{A}+\mathbb{A}^{2}$. Then one computes

$$
Q(\mathbb{A}+\mathbb{B})=Q(\mathbb{A})+Q(\mathbb{B})+[\mathbb{A}, \mathbb{B}],
$$

where $[\mathbb{A}, \mathbb{B}]=\mathbb{A} \mathbb{B}+\mathbb{B} \mathbb{A}$. Thus $Q$ is a quadratic map with associated bilinear map equal to the Lie bracket $[\cdot, \cdot]$. We denote this quadratic map by $Q_{\mathfrak{g l}_{n}}$.

Let $K_{n}\left(\mathbb{Z} / 2^{m}\right)$ be the kernel of the map $G L_{n}\left(\mathbb{Z} / 2^{m}\right) \rightarrow G L_{n}(\mathbb{Z} / 2)$ defined by the mod 2 reduction of the entries of the given matrix. Observe that

$$
K_{n}(\mathbb{Z} / 8)=\left\{\mathbb{I}+2 \mathbb{A} \mid \mathbb{A} \in \mathfrak{g l}_{n}(\mathbb{Z} / 4)\right\}
$$

is a non-abelian group of exponent 4 . Consider the mod 4 reduction map

$$
\varphi: K_{n}(\mathbb{Z} / 8) \rightarrow K_{n}(\mathbb{Z} / 4) .
$$

Note that both $K_{n}(\mathbb{Z} / 4)$ and $\operatorname{Ker}(\varphi)$ are isomorphic to $\mathfrak{g l}_{n}\left(\mathbb{F}_{2}\right)$, and that $\operatorname{ker} \varphi$ is a central subgroup of $K_{n}(\mathbb{Z} / 8)$. So, we get a central extension of the form

$$
E: 0 \rightarrow \mathfrak{g l}_{n}\left(\mathbb{F}_{2}\right) \rightarrow K_{n}(\mathbb{Z} / 8) \rightarrow \mathfrak{g l}_{n}\left(\mathbb{F}_{2}\right) \rightarrow 0
$$


Note that $(\mathbb{I}+2 \mathbb{A})^{2}=\mathbb{I}+4\left(\mathbb{A}+\mathbb{A}^{2}\right)$, and so the associated $Q$ for this extension is indeed equal to $Q_{\mathfrak{g l}_{n}}$.

Example 2.6. If $W$ is an $\mathbb{F}_{2}$-subspace of $\mathfrak{g l}_{n}\left(\mathbb{F}_{2}\right)$ such that $\mathbb{A} \in W$ implies $\mathbb{A}^{2} \in W$, then $Q_{\mathfrak{g l}_{n}}$ induces a quadratic map $Q: W \rightarrow W$. We call such a subspace $W$ squareclosed. Since $[\mathbb{A}, \mathbb{B}]=Q(\mathbb{A}+\mathbb{B})+Q(\mathbb{A})+Q(\mathbb{B})$ we have that $W$ is automatically a sub-Lie algebra of $\mathfrak{g l}_{n}\left(\mathbb{F}_{2}\right)$. Some examples of subspaces $W$ with this property are:

(a) $W=\mathfrak{s l}_{n}\left(\mathbb{F}_{2}\right)$, the matrices of trace zero.

(b) $W=\mathfrak{u}_{n}\left(\mathbb{F}_{2}\right)$, the strictly upper triangular matrices.

In this case we call the corresponding quadratic map $Q: W \rightarrow W$ a $\mathfrak{g l}$-induced quadratic map.

\section{Bockstein Closed eXtensions}

We start with some definitions.

Definition 3.1. If $q_{1}, \ldots, q_{n}$ are components of $q$ with respect to some basis of $V$ we denote by $I(Q)$ the ideal $\left(q_{1}, \ldots, q_{n}\right)$ in $H^{*}\left(W, \mathbb{F}_{2}\right)$.

It is easy to see that the ideal $I(Q)$ is indeed independent of the basis chosen for $V$, and hence is completely determined by $Q$.

Definition 3.2. We say that the quadratic map $Q: W \rightarrow V$ is Bockstein closed if $I(Q)$ is invariant under the Bockstein operator on $H^{*}(W, \mathbb{Z} / 2)$. An extension $E(Q): 0 \rightarrow V \rightarrow G(Q) \rightarrow W \rightarrow 0$ is called Bockstein closed if the associated quadratic map $Q$ is Bockstein closed.

Since $I(Q)$ is an ideal generated by homogeneous polynomials of degree 2 , it is closed under the higher Steenrod operations, so $I(Q)$ is a Steenrod closed ideal. We will later use this fact in Section 7 when we are studying effective extensions.

The main examples of Bockstein closed extensions are gl-induced quadratic maps. We will prove the Bockstein closedness of these extensions at the end of this section. We first start with an easy observation.

Proposition 3.3. Let $Q: W \rightarrow V$ be a quadratic map, and let $q \in H^{2}(W, V)$ be the corresponding extension class. Then, $Q$ is Bockstein closed if and only if there is a one dimensional class $L \in H^{1}(W, \operatorname{End}(V))$ such that $\beta(q)=L q$. Here, the multiplication $L q$ is given by the composite

$$
H^{1}(W, \operatorname{End}(V)) \otimes H^{2}(W, V) \stackrel{\cup}{\longrightarrow} H^{3}(W, \operatorname{End}(V) \otimes V) \rightarrow H^{3}(W, V),
$$

where the second map is induced by the evaluation map ev $: \operatorname{End}(V) \otimes V \rightarrow V$, given by $f \otimes u \rightarrow f(u)$.

Proof. Choose a basis $\mathcal{B}_{W}$ for $W$ and $\mathcal{B}_{V}$ for $V$, and let $\left\{x_{1}, \ldots, x_{m}\right\}$ be the dual basis to $\mathcal{B}_{W}$. We can write $q$ as a column vector with entries in $H^{2}\left(W, \mathbb{F}_{2}\right)$. Then, $q$ is Bockstein closed if and only if there is an $n \times n$ matrix $L$ with entries in linear polynomials in $x_{i}$ 's such that $\beta(q)=L q$ where $n=\operatorname{dim}(V)$. Note that $L$ can be considered as an element in $H^{1}\left(W, M_{n \times n}\left(\mathbb{F}_{2}\right)\right)$. Using the basis $\mathcal{B}_{V}$, we can also identify $M_{n \times n}\left(\mathbb{F}_{2}\right)$ with $\operatorname{End}(V)$. So, $L$ can be considered as a class in $H^{1}(W, \operatorname{End}(V))$, and it is easy to see that under these identifications, matrix multiplication $L q$ corresponds to the map given above. 
Definition 3.4. Let $Q: W \rightarrow V$ be a quadratic map, and let $q \in H^{2}(W, V)$ be the corresponding extension class. If $L \in H^{1}(W, \operatorname{End}(V))$ is a class satisfying $\beta(q)=L q$, then we say that $L$ is a binding operator for $Q$. If a specific basis for $V$ is chosen, then $L$ can be represented as a matrix with linear polynomial entries. In this case we call $L$ a binding matrix.

It is clear that one can have many different matrices for the same operator. Any two such matrices will be conjugate to each other by a scalar matrix. On the other hand, one can also have two different binding operators $L_{1}$ and $L_{2}$ for a quadratic map $Q$. The following example shows a case where this happens.

Example 3.5. Let $q=(x y, y z)$. Then, $\beta(q)=(x y(x+y), y z(y+z))$. We can write

or

$$
\left[\begin{array}{l}
\beta\left(q_{1}\right) \\
\beta\left(q_{2}\right)
\end{array}\right]=\left[\begin{array}{cc}
x+y & 0 \\
0 & y+z
\end{array}\right]\left[\begin{array}{l}
q_{1} \\
q_{2}
\end{array}\right]
$$

$$
\left[\begin{array}{c}
\beta\left(q_{1}\right) \\
\beta\left(q_{2}\right)
\end{array}\right]=\left[\begin{array}{cc}
x+y+z & x \\
z & x+y+z
\end{array}\right]\left[\begin{array}{l}
q_{1} \\
q_{2}
\end{array}\right] .
$$

Note that the second binding matrix is not conjugate to a diagonal matrix. So, there exists more than one binding operator in this case. This can happen because the ideal $\left(q_{1}, q_{2}\right)$ is not a free $k[x, y, z]$-module over the generators $\left\{q_{1}, q_{2}\right\}$. We will see later that under stronger conditions there is a unique binding operator $L$ for $q$.

The following is a reformulation of Proposition 3.3, which is quite useful in many instances.

Theorem 3.6. Let $Q: W \rightarrow V$ be a quadratic map, and let $B$ be the bilinear map associated to $Q$. Then, $Q$ is Bockstein closed if and only if there exists a bilinear map $P: V \times W \rightarrow V$ which satisfies the identity

$$
P(Q(x), y)=B(x, y)+P(B(x, y), x)
$$

for all $x, y \in W$.

Before the proof we first do some calculations. Let $\left\{v_{1}, \ldots, v_{n}\right\}$ be a basis for $V$, and let $\left\{w_{1}, \ldots, w_{m}\right\}$ be a basis for $W$ with dual basis $\left\{x_{1}, \ldots, x_{m}\right\}$. As usual we write $q=\left(q_{1}, \ldots, q_{n}\right)$ for the extension class $q \in H^{2}(W, V)$ associated to $Q$. Note that by Proposition 2.2, for each $k=1, \ldots, n$, we have

$$
q_{k}=\sum_{i} Q_{k}(i) x_{i}^{2}+\sum_{i<j} B_{k}(i, j) x_{i} x_{j}
$$

where $Q_{k}(i)=Q_{k}\left(w_{i}\right)$ and $B_{k}(i, j)=B_{k}\left(w_{i}, w_{j}\right)$. Applying the Bockstein map we get

$$
\beta\left(q_{k}\right)=\sum_{i<j} B_{k}(i, j)\left(x_{i}^{2} x_{j}+x_{i} x_{j}^{2}\right)=\sum_{i, j} B_{k}(i, j) x_{i}^{2} x_{j} .
$$

Given an element $L \in H^{1}(W, \operatorname{End}(V))$, we can consider it as a homomorphism $L: W \rightarrow \operatorname{End}(V)$ via the isomorphism

$$
H^{1}(W, \operatorname{End}(V)) \cong \operatorname{Hom}(W, \operatorname{End}(V)) .
$$

This allows us to describe a correspondence between classes $L \in H^{1}(W, \operatorname{End}(V))$ and bilinear maps $P: V \times W \rightarrow V$. The correspondence is given by $P(v, w)=$ $L(w)(v)$ for all $v \in V$ and $w \in W$. Therefore, if $L$ denotes the matrix for an element 
in $H^{1}(W, \operatorname{End}(V))$ with respect to the above choice of basis for $V$ and $W$, then we can write

$$
L_{k s}=\sum_{p} P_{k}(s, p) x_{p},
$$

where $P_{k}(s, p)$ is short for $P_{k}\left(v_{s}, w_{p}\right)$ and $P_{k}$ is the $k$-th coordinate of $P$. We have the following:

Lemma 3.7. The equation $\beta(q)=L q$ holds if and only if the following two equations hold:

$$
\begin{gathered}
P\left(Q\left(w_{i}\right), w_{j}\right)=B\left(w_{i}, w_{j}\right)+P\left(B\left(w_{i}, w_{j}\right), w_{i}\right) \\
P\left(B\left(w_{i}, w_{j}\right), w_{k}\right)+P\left(B\left(w_{j}, w_{k}\right), w_{i}\right)+P\left(B\left(w_{k}, w_{i}\right), w_{j}\right)=0
\end{gathered}
$$

for all $i, j, k \in\{1, \ldots, n\}$.

Proof. First note that by Equation 2 above, we have

$$
\beta\left(q_{k}\right)=\sum_{i, j} B_{k}(i, j) x_{i}^{2} x_{j}
$$

for all $k$. On the other hand, the $k$-th entry of $L q$ is equal to $\sum_{s} L_{k s} q_{s}$. Writing this sum in detail, we get

$$
\begin{aligned}
\sum_{s} L_{k s} q_{s} & =\sum_{s}\left[\left(\sum_{p} P_{k}(s, p) x_{p}\right)\left(\sum_{i} Q_{s}(i) x_{i}^{2}+\sum_{i<j} B_{s}(i, j) x_{i} x_{j}\right)\right] \\
& =\sum_{s} \sum_{p, i} Q_{s}(i) P_{k}(s, p) x_{i}^{2} x_{p}+\sum_{s} \sum_{p, i<j} B_{s}(i, j) P_{k}(s, p) x_{p} x_{i} x_{j} \\
& =\sum_{p, i}\left(\sum_{s} Q_{s}(i) P_{k}(s, p)\right) x_{i}^{2} x_{p}+\sum_{p, i<j}\left(\sum_{s} B_{s}(i, j) P_{k}(s, p)\right) x_{p} x_{i} x_{j} \\
& =\sum_{i, j}\left(\sum_{s} Q_{s}(i) P_{k}(s, j)\right) x_{i}^{2} x_{j}+\sum_{p, i<j}\left(\sum_{s} B_{s}(i, j) P_{k}(s, p)\right) x_{p} x_{i} x_{j} .
\end{aligned}
$$

The second term on the righthand side (SRHS) can be manipulated more:

$$
\begin{aligned}
S L H S & =\sum_{p=i<j}\left(\sum_{s} B_{s}(i, j) P_{k}(s, i)\right) x_{i}^{2} x_{j}+\sum_{i<j=p}\left(\sum_{s} B_{s}(i, j) P_{k}(s, j)\right) x_{i} x_{j}^{2} \\
& +\sum_{\substack{i<j \\
p \neq i, j}}\left(\sum_{s} B_{s}(i, j) P_{k}(s, p)\right) x_{p} x_{i} x_{j} \\
& =\sum_{i, j}\left(\sum_{s} B_{s}(i, j) P_{k}(s, i)\right) x_{i}^{2} x_{j}+\sum_{\substack{i<j \\
p \neq i, j}}\left(\sum_{s} B_{s}(i, j) P_{k}(s, p)\right) x_{p} x_{i} x_{j},
\end{aligned}
$$

where the last line follows from the identity $B_{s}(i, j)=B_{s}(j, i)$. Putting these equations together, we get

$$
\begin{aligned}
\beta\left(q_{k}\right)+\sum_{s} L_{k s} q_{s} & =\sum_{i, j}\left(B_{k}(i, j)+\sum_{s} Q_{s}(i) P_{k}(s, j)+\sum_{s} B_{s}(i, j) P_{k}(s, i)\right) x_{i}^{2} x_{j} \\
& +\sum_{\substack{i<j \\
p \neq i, j}}\left(\sum_{s} B_{s}(i, j) P_{k}(s, p)\right) x_{p} x_{i} x_{j} .
\end{aligned}
$$


Fixing an order for $\{p, i, j\}$, the second summand in the above equation becomes

$$
\sum_{p<i<j}\left[\sum_{s}\left(B_{s}(i, j) P_{k}(s, p)+B_{s}(p, j) P_{k}(s, i)+B_{s}(p, i) P_{k}(s, j)\right)\right] x_{p} x_{i} x_{j} .
$$

So, we obtain that $\beta(q)+L q=0$ if and only if

$$
B_{k}(i, j)+\sum_{s} Q_{s}(i) P_{k}(s, j)+\sum_{s} B_{s}(i, j) P_{k}(s, i)=0
$$

and

$$
\sum_{s}\left(B_{s}(i, j) P_{k}(s, p)+B_{s}(p, j) P_{k}(s, i)+B_{s}(p, i) P_{k}(s, j)\right)=0
$$

hold for all $i, j, k$. It is easy to see that these equations are the same as Equations (3) and (4).

We will also need the following lemma:

Lemma 3.8. If $P: V \times W \rightarrow V$ is a bilinear form satisfying the identity

$$
P(Q(x), y)=B(x, y)+P(B(x, y), x)
$$

for all $x, y \in W$, then it satisfies

$$
P(B(x, y), z)+P(B(y, z), x)+P(B(z, x), y)=0
$$

for all $x, y, z \in W$.

Proof. We show this by a direct calculation:

$$
\begin{aligned}
P(B(x, y), z)= & P(Q(x)+Q(y)+Q(x+y), z) \\
= & P(Q(x), z)+P(Q(y), z)+P(Q(x+y), z) \\
= & B(x, z)+P(B(x, z), x)+B(y, z)+P(B(y, z), y) \\
& +B(x+y, z)+P(B(x+y, z), x+y) \\
= & P(B(x, z), x)+P(B(y, z), y)+P(B(x+y, z), x+y) \\
= & P(B(x, z), x)+P(B(y, z), y)+P(B(x, z)+B(y, z), x+y) \\
= & P(B(x, z), y)+P(B(y, z), x) \\
= & P(B(y, z), x)+P(B(z, x), y) .
\end{aligned}
$$

Now, we are ready to prove Theorem 3.6.

Proof of Theorem [3.6. First assume that there is a bilinear map $P: V \times W \rightarrow V$ satisfying the identity $P(Q(x), y)=B(x, y)+P(B(x, y), x)$ for all $x, y \in W$. Let $L$ be the associated cohomology class. By Lemma 3.7, we just need to show that the bilinear map $P$ satisfies Equations (3) and (4). For the first equation take $x=w_{i}$ and $y=w_{j}$, and consider the $k$-th coordinates. To get the second equation, we first use Lemma 3.8, and put $x=w_{i}, y=w_{j}$ and $z=w_{p}$ in Equation (5).

For the converse, assume that there is a binding operator $L \in H^{1}(W, \operatorname{End}(V))$ satisfying the equation $\beta(q)=L q$. Let $P: V \times W \rightarrow V$ be the bilinear map associated to $L$. Lemma 3.7 gives us that $P$ satisfies Equations (3) and (4) for all $i, j, k \in\{1, \ldots, n\}$. By linearity this implies that the equation

$$
P(Q(x), y)=B(x, y)+P(B(x, y), x)
$$

is satisfied for all $x, y \in W$. Thus the proof is complete. 
As an immediate corollary of Theorem 3.6, we obtain

Corollary 3.9. Any $\mathfrak{g l}$-induced quadratic map $Q: W \rightarrow W$ is Bockstein closed.

Proof. For $Q: \mathfrak{g l}_{n}\left(\mathbb{F}_{2}\right) \rightarrow \mathfrak{g l}_{n}\left(\mathbb{F}_{2}\right)$ we can set $P(\mathbb{A}, \mathbb{B})=[\mathbb{A}, \mathbb{B}]$. Then we compute

$$
\begin{aligned}
P(Q(\mathbb{A}), \mathbb{B}) & =\left[\mathbb{A}+\mathbb{A}^{2}, \mathbb{B}\right] \\
& =[\mathbb{A}, \mathbb{B}]+\left[\mathbb{A}^{2}, \mathbb{B}\right] \\
& =[\mathbb{A}, \mathbb{B}]+[[\mathbb{A}, \mathbb{B}], \mathbb{A}] .
\end{aligned}
$$

Thus the equation in Theorem 3.6 holds with $P=B$, and hence the $Q_{\mathfrak{g l}_{n}}$ is Bockstein closed. It is easy to see that $P: W \times W \rightarrow W$ for any square-closed subspace $W$, and so every $\mathfrak{g l}$-induced $Q: W \rightarrow W$ is Bockstein closed.

\section{UNIFORM LIFTING AND BINDING OPERATORS}

Throughout this section $Q: W \rightarrow V$ will denote an arbitrary quadratic form, and $E(Q): 0 \rightarrow V \rightarrow G(Q) \rightarrow W \rightarrow 0$ will be the central extension associated to $Q$. As usual we will denote the extension class of this extension by $q$.

Definition 4.1. Let $M$ be a $\mathbb{Z}[W]$-module such that $M / 2 M \cong V$ as $\mathbb{Z} / 2[W]$ modules. We say that $E(Q)$ lifts to an extension with kernel $M$ if there is an extension of the form

$$
\widetilde{E}(Q): 0 \rightarrow M \rightarrow \widetilde{G}(Q) \rightarrow W \rightarrow 0
$$

such that the following diagram commutes:

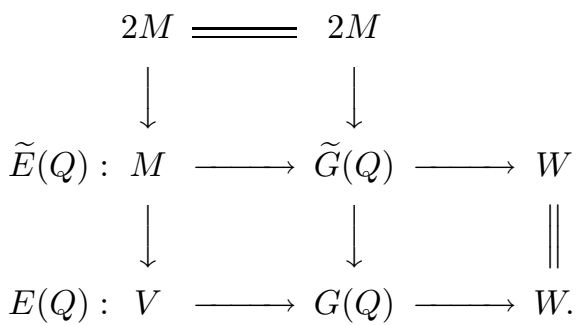

In the case that $M$ is a $\mathbb{Z} / 4$-free $\mathbb{Z} / 4[W]$-module $(\mathbb{Z} / 4[W]$-lattice $)$, we say that $E(Q)$ has a uniform lifting. If $M$ is a $\mathbb{Z}$-free $\mathbb{Z}[W]$-module $(\mathbb{Z}[W]$-lattice $)$, then we say that $E$ has a uniform integral lifting.

It is well known that a quadratic map $Q$ is Bockstein closed if and only if the associated extension $E(Q)$ has a uniform lifting. However, the known proofs of this statement do not provide an explicit connection between the quadratic form and the $\mathbb{Z} / 4[W]$-lattice structure of $M$. In this section we introduce the concept of binding operators and find a direct way to calculate the module structure of $M$ from the binding operator $L$.

We start with the following observation:

Lemma 4.2. Suppose that $M$ is a $\mathbb{Z} / 4[W]$-lattice such that $2 M$ and $M / 2 M$ are trivial $\mathbb{Z} / 2[W]$-modules isomorphic to $V$. Then, $E(Q)$ lifts to an extension with kernel $M$ if and only if $\delta(q)=0$, where $\delta: H^{2}(W, V) \rightarrow H^{3}(W, V)$ is the connecting homomorphism of the long exact sequence for the coefficient sequence $0 \rightarrow V \rightarrow$ $M \rightarrow V \rightarrow 0$ of $\mathbb{Z}[W]$-modules. 
Proof. Recall that the long exact sequence for the extension $0 \rightarrow V \rightarrow M \rightarrow V \rightarrow 0$ is of the form

$$
\cdots \rightarrow H^{2}(W, M) \rightarrow H^{2}(W, V) \stackrel{\delta}{\longrightarrow} H^{3}(W, V) \rightarrow \cdots .
$$

So, from this it is clear that $q$ lifts to a class $\tilde{q} \in H^{2}(W, M)$ if and only if $\delta(q)=0$. Lifting the extension class is equivalent to lifting the associated extension, so the proof of the lemma is complete.

So, it remains to understand the boundary homomorphism for the extension $0 \rightarrow V \rightarrow M \rightarrow V \rightarrow 0$. For this we look at the boundary homomorphism on the chain level. We will consider the boundary operators associated to sequences of $\mathbb{Z}[W]$-modules $0 \rightarrow A \rightarrow B \rightarrow C \rightarrow 0$ where $A$ and $C$ have trivial $\mathbb{Z}[W]$-module structure. To do this we will discuss the notions of binding functions and binding operators.

Let $0 \rightarrow A \rightarrow B \stackrel{\pi}{\rightarrow} C \rightarrow 0$ be a short exact sequence of abelian groups. Let $G L(B ; A, C)$ be the group of automorphisms of $B$ which induce the identity map on $A$ and $C$.

Lemma 4.3. There are isomorphisms $\log : G L(B ; A, C) \rightarrow \operatorname{Hom}(C, A)$ and $\exp :$ $\operatorname{Hom}(C, A) \rightarrow G L(B ; A, C)$ (inverse to each other) given by $\log (f)(c)=f(\hat{c})-\hat{c}$ for all $c \in C$ where $\hat{c} \in B$ has $\pi(\hat{c})=c$, and $\exp (\mu)(b)=b+\mu(\pi(b))$ for all $b \in B$.

Proof. We first show that $\log$ and exp are well-defined functions. Note that if $f \in G L(B ; A, C)$, then $f(\hat{c})-\hat{c}$ projects trivially under $\pi$ since $f$ induces the identity map on $C$ and hence $f(\hat{c})-\hat{c} \in A$. Also since $f$ induces the identity map on $A, f(\hat{c})-\hat{c}$ is indeed independent of the lift $\hat{c}$ of $c$. Finally $\log (f)(c+d)=$ $f(\hat{c}+\hat{d})-(\hat{c}+\hat{d})=\log (f)(c)+\log (f)(d)$ since $f$ is a homomorphism. Thus $\log (f) \in \operatorname{Hom}(C, A)$.

It is clear that $\exp (\mu)$ defines an endomorphism of $G$ which induces the identity map on $A$ and $C$. Since $\exp (-\mu)$ is easily seen to be its inverse, $\exp (\mu)$ is an element of $G L(B ; A, C)$.

Finally since it is easy to see that exp and $\log$ are inverse functions, to show they are isomorphisms, we need only check that $\log$ is a homomorphism. If $f, g \in$ $G L(B ; A, C)$, then

$$
\begin{aligned}
\log (f \circ g)(c) & =f(g(\hat{c}))-\hat{c} \\
& =f(g(\hat{c}))-g(\hat{c})+g(\hat{c})-\hat{c} \\
& =\log (f)(c)+\log (g)(c),
\end{aligned}
$$

where the final step follows since $g(\hat{c})$ is also a lift of $c$ since $g$ induces the identity map on $C$. Thus $\log (f \circ g)=\log (f)+\log (g)$ and the proof is complete.

Now given a sequence of $\mathbb{Z}[W]$-modules $0 \rightarrow A \rightarrow B \rightarrow C \rightarrow 0$ such that $A$ and $C$ are trivial $\mathbb{Z}[W]$-modules, it is clear that we get a representation $\rho_{B}: W \rightarrow$ $G L(B ; A, C)$. Thus we obtain a homomorphism $\log \left(\rho_{B}\right): W \rightarrow \operatorname{Hom}(C, A)$ where $\log \left(\rho_{B}\right)=\log \circ \rho_{B}$.

The extension above yields a long exact sequence whose boundary operator is $\delta: H^{*}(W, C) \rightarrow H^{*+1}(W, A)$. On the other hand we may also consider the sequence $0 \rightarrow A \rightarrow B \rightarrow C \rightarrow 0$ as a sequence of trivial $\mathbb{Z}[W]$-modules, and this will yield another long exact sequence with boundary operator $\delta_{\text {triv }}: H^{*}(W, C) \rightarrow$ $H^{*+1}(W, A)$. 
In general $\delta$ will be different from $\delta_{\text {triv }}$ due to the "twisting", i.e., nontrivial action of $W$ on $B$. The next proposition makes the connection between these two boundary operators explicit.

Proposition 4.4. Let $0 \rightarrow A \rightarrow B \rightarrow C \rightarrow 0$ be a short exact sequence of $W$ modules such that $A$ and $C$ are trivial $W$-modules. Let $\delta: H^{*}(W, C) \rightarrow H^{*+1}(W, A)$ be the boundary operator associated to this sequence and $\delta_{\text {triv }}: H^{*}(W, C) \rightarrow$ $H^{*+1}(W, A)$ be the boundary operator associated with the same sequence and trivial $W$-action. Then,

$$
\delta=\delta_{\text {triv }}+\mathbb{M}
$$

where $\mathbb{M}$ can be defined on cochains $\mathbb{M}: C^{n}(W, C) \rightarrow C^{n+1}(W, A)$ as follows:

$$
\mathbb{M}(f)\left(z_{0}, \ldots, z_{n}\right)=\log \left(\rho_{B}\left(z_{0}\right)\right)\left(f\left(z_{1}, \ldots, z_{n}\right)\right)
$$

for all $f \in C^{n}(W, C)$. We will call $\mathbb{M}: H^{n}(W, C) \rightarrow H^{n+1}(W, A)$ the binding operator of the extension.

Proof. Consider normalized standard bar resolutions. The exact sequence $0 \rightarrow$ $A \rightarrow B \rightarrow C \rightarrow 0$ induces a commuting diagram

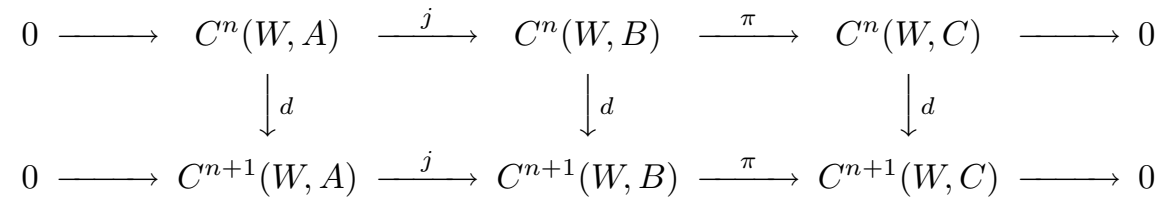

and connection homomorphism $\delta: C^{n}(W, C) \rightarrow C^{n+1}(W, A)$ defined as follows: For $f \in C^{n}(W, C)$, we lift (valuewise) to get $\hat{f} \in C^{n}(W, B)$. Then $\delta(f)=d \hat{f}$. Using $\rho_{B}: W \rightarrow G L(B ; A, C)$ to explicitly write the $W$ action on $B$ and computing we get:

$$
\begin{aligned}
(\delta f)\left(z_{0}, \ldots, z_{n}\right) & =\rho_{B}\left(z_{0}\right)\left(\hat{f}\left(z_{1}, \ldots, z_{n}\right)\right) \\
& -\hat{f}\left(z_{0} z_{1}, z_{2}, \ldots, z_{n}\right) \\
& \ldots \\
& \pm \hat{f}\left(z_{0}, z_{1}, \ldots, z_{n-1} z_{n}\right) \\
& \mp \hat{f}\left(z_{0}, z_{1}, \ldots, z_{n-1}\right),
\end{aligned}
$$

which we write as

$$
\begin{aligned}
(\delta f)\left(z_{0}, \ldots, z_{n}\right) & =\rho_{B}\left(z_{0}\right)\left(\hat{f}\left(z_{1}, \ldots, z_{n}\right)\right)-\hat{f}\left(z_{1}, \ldots, z_{n}\right)+\left(\delta_{\text {triv }} f\right)\left(z_{0}, \ldots, z_{n}\right) \\
& =\log \left(\rho_{B}\left(z_{0}\right)\right)\left(f\left(z_{1}, \ldots, z_{n}\right)\right)+\left(\delta_{\text {triv }} f\right)\left(z_{0}, \ldots, z_{n}\right) \\
& =\left(\delta_{\text {triv }} f+\mathbb{M} f\right)\left(z_{0}, \ldots, z_{n}\right)
\end{aligned}
$$

where $\delta_{\text {triv }}$ denotes the connection homomorphism corresponding to the sequence $0 \rightarrow A \rightarrow B \rightarrow C \rightarrow 0$ with the trivial $W$-action. Thus we see that $\delta=\delta_{\text {triv }}+\mathbb{M}$ on cochains. Since the general theory tells us that $\delta$ and $\delta_{\text {triv }}$ give well defined homomorphisms $H^{n}(W, C) \rightarrow H^{n}(W, A)$, we see that $\mathbb{M}$ does also. The proof is complete.

For computational purposes a more explicit form for $\mathbb{M}$ is desirable. To get this, note that the general cup product construction gives us a map

$$
H^{1}(W, \operatorname{Hom}(C, A)) \otimes H^{n}(W, C) \stackrel{\cup}{\rightarrow} H^{n+1}(W, \operatorname{Hom}(C, A) \otimes C) .
$$


The composition pairing $\operatorname{Hom}(C, A) \otimes C \rightarrow A$ induces a map

$$
H^{n+1}(W, \operatorname{Hom}(C, A) \otimes C) \rightarrow H^{n+1}(W, A),
$$

which when composed with the cup product above yields a cup product:

$$
H^{1}(W, \operatorname{Hom}(C, A)) \times H^{n}(W, C) \stackrel{\cup}{\rightarrow} H^{n+1}(W, A) .
$$

Finally note that given a representation $\rho_{B}: W \rightarrow G L(B ; A, C)$, we have

$$
\log \left(\rho_{B}\right) \in \operatorname{Hom}(W, \operatorname{Hom}(C, A))=H^{1}(W, \operatorname{Hom}(C, A)) .
$$

Thus taking the cup product with $\log \left(\rho_{B}\right) \in H^{1}(W, \operatorname{Hom}(C, A))$ induces a map

$$
H^{n}(W, C) \rightarrow H^{n+1}(W, A) .
$$

Notice that on cochains we have (see Brown [2, page 110)

$$
\left(\log \left(\rho_{B}\right) \cup f\right)\left(z_{0}, \ldots, z_{n}\right)=(-1)^{n} \log \left(\rho_{B}\left(z_{0}\right)\right) f\left(z_{1}, \ldots, z_{n}\right),
$$

and so we see that the binding operator $\mathbb{M}$ is induced by cup product (from the right) by $\log \left(\rho_{B}\right) \in H^{1}(W, \operatorname{Hom}(C, A))$. Thus it is relatively routine to describe $\mathbb{M}$ in any computational situation. We summarize this in the following proposition:

Proposition 4.5. If $0 \rightarrow A \rightarrow B \rightarrow C \rightarrow 0$ is a short exact sequence of $W$ modules with corresponding representation $\rho_{B}: W \rightarrow G L(B ; A, C)$, then considering $\log \left(\rho_{B}\right) \in H^{1}(W, \operatorname{Hom}(C, A))$ we have $\mathbb{M}(-)=(-) \cup \log \left(\rho_{B}\right)$.

Now, we state our main result of this section.

Theorem 4.6. If $E(Q)$ lifts to an extension with kernel $M$, then $\beta(q)=$ $\left[\log ^{*}\left(\rho_{M}\right)\right] q$. Conversely, if $\beta(q)=L q$ for some $L \in H^{1}(W$, $\operatorname{End}(V))$, then $E(Q)$ lifts to an extension with kernel $M$ where $M$ has the representation $\exp ^{*}(L) \in$ $\operatorname{Hom}(W, G L(M ; V, V))$.

Note that in general $E(Q)$ may have more than one module where the above lifting is possible. This is similar to having more than one $L$ such that $\beta(q)=L q$. Theorem 4.6 says that choosing one fixes the other.

This result has many consequences for the structure of extension classes. We will investigate them further in other sections. We end this section with some calculations to illustrate the effectiveness of the above result.

Example 4.7. Let $W=V=\left(\mathbb{F}_{2}\right)^{3}$ with standard basis and let $E(Q)$ be an extension with extension class

$$
q=\left(x_{1}^{2}+x_{2} x_{3}, x_{2}^{2}+x_{1} x_{2}, x_{3}^{2}+x_{1} x_{3}\right) .
$$

Then, we have

$$
\left[\begin{array}{l}
\beta\left(q_{1}\right) \\
\beta\left(q_{2}\right) \\
\beta\left(q_{3}\right)
\end{array}\right]=\left[\begin{array}{ccc}
0 & x_{3} & x_{2} \\
0 & x_{1} & 0 \\
0 & 0 & x_{1}
\end{array}\right]\left[\begin{array}{l}
q_{1} \\
q_{2} \\
q_{3}
\end{array}\right] .
$$

This shows that $E(Q)$ has a lifting with kernel $M$ where $M$ has representation $\rho: W \rightarrow G L(W ; V, V)$ with

$$
\rho\left(\alpha_{1}, \alpha_{2}, \alpha_{3}\right)=\left[\begin{array}{ccc}
1 & 2 \alpha_{3} & 2 \alpha_{2} \\
0 & 1+2 \alpha_{1} & 0 \\
0 & 0 & 1+2 \alpha_{1}
\end{array}\right] .
$$


Note that $L$ is upper triangular; hence $M$ has a filtration

$$
0 \leq\langle(1,0,0)\rangle \leq\langle(1,0,0),(0,1,0)\rangle \leq M
$$

whose sections are one dimensional $\mathbb{Z} / 4[W]$-lattices.

Example 4.8. Let $W=V=\left(\mathbb{F}_{2}\right)^{3}$ and let $E(Q)$ be an extension with extension class

Then, we have

$$
q=\left(x_{1}^{2}, x_{2}^{2}+x_{1} x_{2}, x_{3}^{2}+x_{2} x_{3}\right)
$$

$$
\left[\begin{array}{l}
\beta\left(q_{1}\right) \\
\beta\left(q_{2}\right) \\
\beta\left(q_{3}\right)
\end{array}\right]=\left[\begin{array}{ccc}
0 & 0 & 0 \\
0 & x_{2} & 0 \\
0 & 0 & x_{3}
\end{array}\right]\left[\begin{array}{l}
q_{1} \\
q_{2} \\
q_{3}
\end{array}\right] .
$$

Thus, $E(Q)$ has a lifting where in this case $M$ has the representation

$$
\rho\left(\alpha_{1}, \alpha_{2}, \alpha_{3}\right)=\left[\begin{array}{ccc}
1 & 0 & 0 \\
0 & 1+2 \alpha_{2} & 0 \\
0 & 0 & 1+2 \alpha_{3}
\end{array}\right] .
$$

In this case $L$ is diagonal; hence $M$ has a decomposition into one dimensional lattices

$$
M \cong\langle(1,0,0)\rangle \oplus\langle(0,1,0)\rangle \oplus\langle(0,0,1)\rangle
$$

Note that, applying the Bockstein operator to the equation $\beta(q)=L q$, one gets that $\left[\beta(L)+L^{2}\right] q=0$. It turns out that the matrix $\beta(L)+L^{2}$ plays an important role. In the first example above, we have

$$
\beta(L)+L^{2}=\left[\begin{array}{ccc}
0 & q_{3} & q_{2} \\
0 & 0 & 0 \\
0 & 0 & 0
\end{array}\right]
$$

whereas we have $\beta(L)+L^{2}=0$ in the second case. We will see in the next section that the matrix $\beta(L)+L^{2}$ is in fact the obstruction for the module $M$ to decompose into a direct sum of one dimensional lattices.

\section{Diagonalizable extensions}

Let $E(Q): 0 \rightarrow V \rightarrow G(Q) \rightarrow W \rightarrow 0$ be a Bockstein closed, central extension corresponding to the quadratic map $Q: W \rightarrow V$. Let $q \in H^{2}(W, V)$ denote the extension class for $E(Q)$.

Definition 5.1. We say that the quadratic map $Q$ is diagonalizable if there exists a basis for $V$ such that the components $q_{1}, \ldots, q_{n}$ are individually Bockstein closed. In other words, for some basis of $V$, we can write $q=\left(q_{1}, \ldots, q_{n}\right)$ where for each $i$ we have $\beta\left(q_{i}\right)=\lambda_{i} q_{i}$ for some linear polynomial $\lambda_{i}$.

It is clear that diagonalizable quadratic maps are Bockstein closed with a diagonal binding matrix $L$. The converse also holds:

Lemma 5.2. Let $Q: W \rightarrow V$ be a Bockstein closed quadratic map with binding matrix $L$ for some basis of $V$. If there is an invertible scalar matrix $N$ such that $N^{-1} L N$ is a diagonal matrix, then $Q$ is diagonalizable.

Proof. Let $q^{\prime}=N^{-1} q$. Then,

$$
\beta\left(q^{\prime}\right)=N^{-1} \beta(q)=N^{-1} L q=\left(N^{-1} L N\right) q^{\prime}
$$

shows that the coordinates of $q^{\prime}$ are individually Bockstein closed. 
When there is an invertible scalar matrix $N$ such that $N^{-1} L N$ is a diagonal matrix, we say that $L$ is diagonalizable. We have the following useful criteria for the diagonalizability of $L$.

Lemma 5.3. Let $L$ be a matrix with linear polynomial entries over the field $\mathbb{F}_{2}$. Then, $L$ is diagonalizable with a scalar matrix if and only if $\beta(L)+L^{2}=0$.

Proof. Write $L=\sum_{i} L_{i} x_{i}$. We have

$$
\beta(L)+L^{2}=\sum_{i}\left(L_{i}^{2}+L_{i}\right) x_{i}^{2}+\sum_{i<j}\left(L_{i} L_{j}+L_{j} L_{i}\right) x_{i} x_{j} .
$$

Thus, $\beta(L)+L^{2}=0$ if and only if $L_{i}\left(L_{i}+I\right)=0$ for all $i$, and $L_{i} L_{j}=L_{j} L_{i}$ for all $i, j$. Over the field $\mathbb{F}_{2}$, a family $\left\{L_{i}\right\}$ is simultaneously diagonalizable if and only if these equations are satisfied. Note that if $N$ is the matrix that simultaneously diagonalizes the family $\left\{L_{i}\right\}$, then $N$ diagonalizes $L$ as well.

The argument used in the above proof is due to Dave Rusin. He uses this argument to prove Lemma 20 in [6] which states that all Bockstein closed extensions are diagonalizable. However, this lemma is not correct. There are Bockstein closed extensions which are not diagonalizable. The following is an example of such an extension.

Example 5.4. Let $E$ be an extension with $q=\left(x^{2}+y z, y^{2}+x z, z^{2}\right)$. Then

$$
\beta(q)=(y z(y+z), x z(x+z), 0),
$$

and we can write

$$
\left[\begin{array}{c}
\beta\left(q_{1}\right) \\
\beta\left(q_{2}\right) \\
\beta\left(q_{3}\right)
\end{array}\right]=\left[\begin{array}{ccc}
0 & z & x+y \\
z & 0 & x+y \\
0 & 0 & 0
\end{array}\right]\left[\begin{array}{l}
q_{1} \\
q_{2} \\
q_{3}
\end{array}\right] .
$$

So the equation $\beta(q)=L q$ holds when $L$ is taken as the above coefficient matrix. In particular, $E$ is a Bockstein closed extension. When we calculate $\beta(L)+L^{2}$, we get

$$
\beta(L)+L^{2}=\left[\begin{array}{ccc}
z^{2} & z^{2} & (x+y)(x+y+z) \\
z^{2} & z^{2} & (x+y)(x+y+z) \\
0 & 0 & 0
\end{array}\right] \neq 0,
$$

so $L$ is not diagonalizable. This also implies that $E$ is not diagonalizable since in this case the binding operator $L$ is unique. Note that if $L^{\prime}$ is another binding matrix satisfying $\beta(q)=L^{\prime} q$, then we would have $L=L^{\prime}$ since the components

$$
x^{2}+y z, y^{2}+x z, z^{2}
$$

of $q$ form a regular sequence. We conclude that $E$ is a Bockstein closed extension which is not diagonalizable. $L$.

Now, we continue to find more equivalent conditions for the diagonalizability of

Proposition 5.5. Let $L=\log \rho_{M} \in H^{1}(W, \operatorname{End}(V))$, and let $M$ be a $\mathbb{Z} / 4[W]$ lattice with representation $\rho_{M} \in G L(M ; V, V)$. Then, the following are equivalent:

(i) $L$ is diagonalizable, i.e., $\beta(L)+L^{2}=0$;

(ii) $M$ is a direct sum of one-dimensional $\mathbb{Z} / 4[W]$-lattices;

(iii) $M$ lifts to a $\mathbb{Z}[W]$-lattice;

(iv) $M$ lifts to a $\mathbb{Z} / 8[W]$-lattice. 
Proof. $(i) \Leftrightarrow(i i)$ follows from the fact that the representation $\rho: W \rightarrow G L(M)$ of $M$ is defined as $\rho(w)=1+2 L(w) \bmod 2$. To see that $(i i) \Rightarrow(i i i)$, note that we just need to show that every one dimensional $\mathbb{Z} / 4[W]$-lattice lifts to an integral lattice. This follows from the fact that the unit group of $\mathbb{Z} / 4$ is $\{ \pm 1\}$ which is also the unit group of $\mathbb{Z}$. The implication (iii) $\Rightarrow(i v)$ is obvious. To complete the proof, we will show that $(i v) \Rightarrow(i)$.

Let $\tilde{\rho}: W \rightarrow G L(\widetilde{M})$ denote the lifting of $\rho$. We can write $\tilde{\rho}(w)=I+2 L(w)+$ $4 a(w) \bmod 8$ for some $a: W \rightarrow \operatorname{End}(V)$. Since $2 w=0$, we have

$$
I=[\tilde{\rho}(w)]^{2}=I+4\left[L(w)^{2}+L(w)\right] \bmod 8 ;
$$

hence $L(w)^{2}+L(w)=0 \bmod 2$ for every $w \in W$. This gives $B L+L^{2}=0$.

As a consequence we obtain the following interesting result:

Proposition 5.6. Let $E^{\prime}: 0 \rightarrow M \rightarrow \Gamma \rightarrow W \rightarrow 0$ be an extension with $M$ a $\mathbb{Z}$-free $\mathbb{Z}[W]$-module such that $M / 2 M$ is a trivial $\mathbb{Z} / 2[W]$-module. Then, the extension

$$
E: 0 \rightarrow M / 2 M \rightarrow \Gamma / 2 M \rightarrow W \rightarrow 0
$$

is a diagonalizable extension.

Proof. Let $Q$ be the quadratic map for the extension $E$. Since $E$ has a lifting

$$
E^{\prime \prime}: 0 \rightarrow M / 4 M \rightarrow \Gamma / 4 M \rightarrow W \rightarrow 0,
$$

it is a Bockstein closed extension whose binding operator $L$ is determined by $M / 4 M$. Since the $\mathbb{Z} / 4[W]$-lattice $M / 4 M$ is reduced from an integral module, by Proposition 5.5. $L$ is diagonalizable, so $Q$ is diagonalizable as well.

The converse of Proposition 5.6 is also known to be true:

Proposition 5.7. Let $E: 0 \rightarrow V \rightarrow G \rightarrow W \rightarrow 0$ be a diagonalizable extension. Then, it lifts to a (unique) extension $\widetilde{E}: 0 \rightarrow M \rightarrow \widetilde{G} \rightarrow W \rightarrow 0$ where $M$ is a $\mathbb{Z}$-free $\mathbb{Z}[W]$-module.

To prove this we will need the following lemma:

Lemma 5.8. Let $q$ be a quadratic polynomial in $m$ variables viewed as an element in $H^{2}\left(W, \mathbb{F}_{2}\right)$ where $m=\operatorname{dim}(W)$ as before. Then $q$ is reducible if and only if $\beta(q)=\ell q$ for some linear polynomial $\ell \in H^{1}\left(W, \mathbb{F}_{2}\right)$. In this case if $q=u v$, then in fact $\ell=u+v$.

Proof. If $q$ is reducible with $q=u v$, then a simple calculation shows that $\beta(q)=\ell q$ with $\ell=u+v$.

Let us prove the converse; so assume $\beta(q)=\ell q$ for some linear polynomial $\ell$. We will show that this implies that $q$ is decomposable. If $m=1$ there is nothing to show, so assume $m>1$.

Case 1: $\ell=0$ : In this case, $\beta(q)=0$ and so $q=\beta(u)=u^{2}$ by the fact that $H^{*}\left(W, \mathbb{F}_{2}\right)$ is $\beta$-acyclic.

Case 2: $\ell \neq 0$ : In this case as $\ell$ is nonzero in $H^{1}\left(W, \mathbb{F}_{2}\right)=\operatorname{Hom}\left(W, \mathbb{F}_{2}\right)$ we can let $H=\operatorname{ker}(\ell)$ and $H$ will be a hyperplane in $W$. Thus $\beta(q)=0$ (and hence $q$ is a square) when restricted to $H^{*}\left(H, \mathbb{F}_{2}\right)$, and so it follows that $q=u^{2}+\ell v \in H^{*}\left(W, \mathbb{F}_{2}\right)$ where $u, v$ are linear and $u$ is algebraically independent from $\ell$. Applying $\beta$ to both sides of the last equation we get $\ell q=\ell^{2} v+\ell v^{2}$. Canceling $\ell$ we get $q=\ell v+v^{2}=$ $v(\ell+v)$ and so $q$ is decomposable as desired. 
We will now prove Proposition 5.7

Proof. Take a basis for $V$ such that, as a $\mathbb{Z} / 2[W]$-module, $V$ decomposes as $V=$ $\bigoplus_{i=1}^{n} V_{i}$ where $\operatorname{dim}\left(V_{i}\right)=1$. Taking components of the extension class $q$ with respect to this basis, say $\left\{q_{1}, \ldots, q_{n}\right\}$, we find that $q_{i}$ represents an extension

$$
0 \rightarrow V_{i} \rightarrow G_{i} \rightarrow W \rightarrow 0
$$

and the $q_{i}$ are individually diagonal, i.e., $\beta\left(q_{i}\right)=\ell_{i} q_{i}$.

Suppose that we can uniformly lift the corresponding extensions for the individual $q_{i}$, say, to extensions

$$
0 \rightarrow M_{i} \rightarrow \widetilde{G}_{i} \rightarrow W \rightarrow 0
$$

with extension class $\tilde{q}_{i}$, where the $M_{i}$ 's are $\mathbb{Z} / 4[W]$-modules with $M_{i} / 2 M_{i}=V_{i}$ and $M_{i} \cong \mathbb{Z} / 4$ as abelian groups. The isomorphism

$$
H^{2}\left(W, \bigoplus_{i=1}^{n} M_{i}\right) \cong \bigoplus_{i=1}^{n} H^{2}\left(W, M_{i}\right)
$$

shows that the $\hat{q}_{i}$ would fit together to give a uniform lift $\tilde{q}=\left(\tilde{q}_{1}, \ldots, \tilde{q}_{n}\right)$ with lifting module $M=\bigoplus_{i=1}^{n} M_{i}$. Thus it is sufficient to prove the theorem in the case $\operatorname{dim}(V)=1$.

Since $\beta(q)=\ell q$ by assumption, Lemma 5.8 gives that $q=u v$ for some 1dimensional classes $u, v$. There are then two cases:

Case 1: $\{u, v\}$ are linearly dependent. In this case $G$ is abelian and a uniform integral lift certainly exists.

Case 2: $\{u, v\}$ are linearly independent. In this case it is easy to check that $G=D_{8} \times S$ where $S$ is elementary abelian with $\operatorname{dim}(S)=\operatorname{dim}(W)-2$ and $V=$ $\left[D_{8}, D_{8}\right]$. This extension has an integral lift (unique) given by $\widetilde{G}=D_{\infty} \times S$ and $M=\left[D_{\infty}, D_{\infty}\right]$ where $D_{\infty}$ is the infinite dihedral group given by a semidirect product of $\mathbb{Z}$ by $\mathbb{Z} / 2$ with twisting given by the sign map.

We end this section with a result which summarizes the results obtained about diagonalizability.

Theorem 5.9. Let $Q: W \rightarrow V$ be a Bockstein closed quadratic map, and let $E: 0 \rightarrow V \rightarrow G \rightarrow W \rightarrow 0$ be the central extension associated to $Q$. Let $q$ denote the extension class for $E$. Then, the following are equivalent:

(i) $Q$ is diagonalizable, i.e., there is a basis for $V$ such that the components of $q$ are individually Bockstein closed.

(ii) There is a choice of basis of $V$ such that the components $q_{1}, \ldots, q_{n}$ of $q$ all decompose as $q_{i}=u_{i} v_{i}$ where $u_{i}, v_{i}$ are linear polynomials.

(iii) There exists a diagonalizable $L \in \operatorname{Hom}(W, \operatorname{End}(V))$ such that $\beta(q)=L q$. This is characterized exactly by the equation $\beta(L)+L^{2}=0$.

(iv) E lifts to an extension with kernel $M$ where $M$ is a direct sum of one dimensional $\mathbb{Z} / 4[W]$-lattices.

(v) E lifts to an extension with kernel $M$ where $M$ is a $\mathbb{Z} / 8[W]$-lattice.

(vi) E lifts to an extension with kernel $M$ where $M$ is a $\mathbb{Z}[W]$-lattice, i.e., E has a uniform integral lifting.

Proof. $(i) \Leftrightarrow($ ii $)$ follows from Lemma 5.8, and Lemma 5.2 gives $(i) \Leftrightarrow(i i i)$. We also have $(i) \Leftrightarrow(v i)$ by Propositions [5.6 and 5.7. Finally, the equivalences $(i v) \Leftrightarrow$ $(v) \Leftrightarrow(v i)$ follow from Proposition 5.5 . 


\section{Triangulable extensions}

As in the previous section, $E(Q): 0 \rightarrow V \rightarrow G(Q) \rightarrow W \rightarrow 0$ denotes an arbitrary Bockstein closed extension associated to a quadratic map $Q: W \rightarrow V$. Let $q \in H^{2}(W, V)$ be the associated extension class.

Definition 6.1. We say that the quadratic map $Q$ is (upper) triangulable if there is a basis for $V$ such that the components $q_{1}, \ldots, q_{n}$ of $q$ have the property that for each $i=1, \ldots, n$, the ideal $\left(q_{i}, q_{i+1}, \ldots, q_{n}\right)$ is a Bockstein closed ideal.

Note that if $Q$ is triangulable, then it is Bockstein closed with an upper triangular binding matrix $L$. The converse also holds: If $Q$ is a quadratic map with a binding matrix $L$ for some basis, then $Q$ is triangulable if there is a scalar matrix $N$ such that $N^{-1} L N$ is an upper triangular matrix. In this case we say that $L$ is triangulable.

From our earlier discussion about the connection between $L$ and the lifting lattice $M$, the following is immediate.

Lemma 6.2. Let $M$ be a $\mathbb{Z} / 4[W]$-lattice with representation $\rho_{M} \in G L(M ; V, V)$, and let $L=\log \rho_{M} \in H^{1}(W, \operatorname{End}(V))$. Then, $L$ is triangulable if and only if $M$ has a filtration $0 \subseteq M_{1} \subseteq M_{2} \subseteq \cdots \subseteq M_{n}=M$ such that each factor $M_{i} / M_{i-1}$ is a rank one $\mathbb{Z} / 4[W]$-lattice.

There are many $\mathbb{Z} / 4[W]$-lattices $M$ which do not have such a filtration even with the extra condition that $M / 2 M$ is a trivial $\mathbb{Z} / 2[W]$-module. Note that given a $\mathbb{Z} / 4[W]$-lattice $M$ such that $V=M / 2 M$ is trivial, there is a homomorphism

$$
L: W \rightarrow \operatorname{End}(V)
$$

associated to it under the exponential-logarithm correspondence. It is easy to see that if $M$ has a filtration with one dimensional factors, then the family $\{L(w) \mid w \in$ $W\}$ is simultaneously triangulable. This means that there is a matrix $N$ such that $N^{-1} L(w) N=T(w)$ is an upper triangular matrix for all $w \in W$. Since $T(w)^{2}+T(w)$ is a strictly upper triangular matrix, it follows that $\left[T(w)^{2}+T(w)\right]^{n}=$ 0 where $n=\operatorname{dim} V$. This implies that $\left[L(w)^{2}+L(w)\right]^{n}=0$ as well. Note that for $w=\alpha_{1} w_{1}+\cdots+\alpha_{m} w_{m}$, the scalar matrix $L(w)^{2}+L(w)$ is equal to the value of $\beta(L)+L^{2}$ calculated by setting $x_{1}=\alpha_{1}, x_{2}=\alpha_{2}, \ldots, x_{n}=\alpha_{n}$. So, if $\left[L(w)^{2}+L(w)\right]^{n}=0$ for all $w \in W$, then we have $\left[\beta(L)+L^{2}\right]^{n}=0$. We have proved the following:

Lemma 6.3. If $L$ is triangulable, then $\beta(L)+L^{2}$ is nilpotent.

Using this we can give an example of a $\mathbb{Z} / 4[W]$-lattice which does not have a filtration:

Example 6.4. Let $m=n=2$ and let $\left\{w_{1}, w_{2}\right\}$ be a basis for $W$. Consider the representation $\rho: W \rightarrow G L_{2}(\mathbb{Z} / 4)$ where

$$
\rho\left(w_{1}\right)=\left[\begin{array}{ll}
1 & 2 \\
0 & 1
\end{array}\right], \quad \rho\left(w_{2}\right)=\left[\begin{array}{ll}
3 & 0 \\
2 & 1
\end{array}\right] .
$$

Then, we have

$$
L=\left[\begin{array}{cc}
x_{2} & x_{1} \\
x_{2} & 0
\end{array}\right] \text { and } \beta(L)+L^{2}=\left[\begin{array}{cc}
x_{1} x_{2} & x_{1}^{2}+x_{1} x_{2} \\
0 & x_{1} x_{2}
\end{array}\right] .
$$

It is clear that $\left[\beta(L)+L^{2}\right]^{k} \neq 0$ for any $k$, so $M$ does not have a one dimensional sublattice. 
In the previous section, we showed that $L$ is diagonalizable if and only if $\beta(L)+$ $L^{2}=0$. So, it is reasonable to ask if the converse of Lemma 6.3 holds. As positive evidence one sees that if $\beta(L)+L^{2}$ is nilpotent, then for every $w \in W$, the operator $L(w)$ is triangulable. This is because, if $\beta(L)+L^{2}$ is nilpotent, i.e., $[\beta(L)+L]^{k}=0$ for some $k$, then

$$
\left[L(w)^{2}+L(w)\right]^{k}=L(w)^{k}[L(w)+I]^{k}=0
$$

holds for every $w \in W$. So, the minimal polynomial of $L(w)$ is a product of linear polynomials, and hence $L(w)$ is triangulable for all $w \in W$ by a standard result in linear algebra. Unfortunately, unless we have an extra structure, in general we do not have simultaneous triangulability. In fact, the following example clearly shows that the converse of Lemma 6.3 fails.

Example 6.5. Let $m=n=2$ and let $\left\{w_{1}, w_{2}\right\}$ be a basis for $W$. Consider the representation $\rho: W \rightarrow \operatorname{Aut}(M)=G L_{2}(\mathbb{Z} / 4)$ where

$$
\rho\left(w_{1}\right)=\left[\begin{array}{ll}
1 & 2 \\
0 & 1
\end{array}\right], \quad \rho\left(w_{2}\right)=\left[\begin{array}{ll}
1 & 0 \\
2 & 1
\end{array}\right] .
$$

Then,

$$
L=\left[\begin{array}{cc}
0 & x_{1} \\
x_{2} & 0
\end{array}\right] \text { and } \beta(L)+L^{2}=\left[\begin{array}{cc}
x_{1} x_{2} & x_{1}^{2} \\
x_{2}^{2} & x_{1} x_{2}
\end{array}\right] .
$$

It is clear that $\left[\beta(L)+L^{2}\right]^{2}=0$. It is easy to check that no nonzero vector is a common $\mathbb{Z} / 4$-eigenvector for $\rho\left(w_{1}\right)$ and $\rho\left(w_{2}\right)$ and thus $M$ has no one dimensional $\mathbb{Z} / 4[W]$-sublattice.

The above examples show that the situation with triangulability is much more complicated. To illustrate that such bad examples also appear as extensions, we calculate $\beta(L)+L^{2}$ for the $\mathfrak{g l}$-induced extension given in Example 2.5,

Example 6.6. Consider the central extension

$$
E\left(Q_{\mathfrak{g l}_{n}}\right): 0 \rightarrow \mathfrak{g l}_{n}\left(\mathbb{F}_{2}\right) \rightarrow G\left(Q_{\mathfrak{g l}_{n}}\right) \rightarrow \mathfrak{g l}_{n}\left(\mathbb{F}_{2}\right) \rightarrow 0
$$

with quadratic map $Q_{\mathfrak{g l}_{n}}(\mathbb{A})=\mathbb{A}+\mathbb{A}^{2}$. Note that we can express the extension class

$$
q \in H^{2}\left(\mathfrak{g l}_{n}\left(\mathbb{F}_{2}\right), \mathfrak{g l}_{n}\left(\mathbb{F}_{2}\right)\right)
$$

as an $n \times n$-matrix $\mathbb{Q}$ whose $i j$-th entry will be the $i j$-th component of $q$. Then one computes

$$
\mathbb{Q}_{i j}(\mathbb{A})=\left(\mathbb{A}^{2}+\mathbb{A}\right)_{i j}=\sum_{k} a_{i k} a_{k j}+a_{i j}
$$

So, we can write $q_{i j}=x_{i j}^{2}+\sum_{k} x_{i k} x_{k j}$, where $x_{i j} \in H^{1}\left(\mathfrak{g l}_{n}\left(\mathbb{F}_{2}\right), \mathbb{Z} / 2\right)$ is the linear form which takes a matrix $\mathbb{A}$ to its $i j$-th entry. Note that if we set

$$
\mathbb{X} \in H^{1}\left(\mathfrak{g l}_{n}\left(\mathbb{F}_{2}\right), \mathfrak{g l}_{n}\left(\mathbb{F}_{2}\right)\right)
$$

as the class corresponding to the identity homomorphism id $: \mathfrak{g l}_{n}\left(\mathbb{F}_{2}\right) \rightarrow \mathfrak{g l}_{n}\left(\mathbb{F}_{2}\right)$, then $\mathbb{X}$ will be a matrix with $i j$-th entry $x_{i j}$, and we will have

$$
\mathbb{Q}=\beta(\mathbb{X})+\mathbb{X}^{2}
$$

Here the ring structure on $H^{*}\left(\mathfrak{g l}_{n}\left(\mathbb{F}_{2}\right), \mathfrak{g l}_{n}\left(\mathbb{F}_{2}\right)\right)$ that we are using is induced from the composition map $\mathfrak{g l}_{n}\left(\mathbb{F}_{2}\right) \times \mathfrak{g l}_{n}\left(\mathbb{F}_{2}\right) \rightarrow \mathfrak{g l}_{n}\left(\mathbb{F}_{2}\right)$ and is noncommutative! 
This gives us an easy way to calculate the image of the Bockstein map from which we obtain

$$
\beta(\mathbb{Q})=\beta(\mathbb{X}) \mathbb{X}+\mathbb{X} \beta(\mathbb{X})=\mathbb{Q} \mathbb{X}+\mathbb{X} \mathbb{Q} .
$$

This shows that for all $(i, j)$ pairs, $\beta\left(q_{i j}\right)$ lies in the ideal generated by the components of $q$ and hence provides another way to see that the quadratic map $Q_{\mathfrak{g l}_{n}}$ is Bockstein closed. Note that the matrix $L$ with respect to some basis can be written as an $n^{2} \times n^{2}$-matrix, but it is much more convenient to think of $L$ as a homomorphism

$$
\left.L: \mathfrak{g l}_{n}\left(\mathbb{F}_{2}\right) \rightarrow \operatorname{End}\left(\mathfrak{g l}_{n}\left(\mathbb{F}_{2}\right)\right)\right)
$$

such that for every $\mathbb{B} \in \mathfrak{g l}_{n}\left(\mathbb{F}_{2}\right)$ the image of $\mathbb{B}$ under $L$ is defined as the endomorphism

$$
L(\mathbb{B}): \mathbb{A} \rightarrow[\mathbb{A}, \mathbb{B}]=\mathbb{A} \mathbb{B}+\mathbb{B} \mathbb{A} .
$$

Using $L(\mathbb{X}): \mathbb{A} \rightarrow[\mathbb{A}, \mathbb{X}]$, we can calculate

$$
\left[\beta(L(\mathbb{X}))+L(\mathbb{X})^{2}\right]: \mathbb{A} \rightarrow\left[\mathbb{A}, \beta(\mathbb{X})+\mathbb{X}^{2}\right]=[\mathbb{A}, \mathbb{Q}] .
$$

This shows, in particular, that

$$
\left[\beta(L)+L^{2}\right] q=\left[\beta(L(\mathbb{X}))+L(\mathbb{X})^{2}\right](\mathbb{Q})=[\mathbb{Q}, \mathbb{Q}]=0,
$$

which we know holds for all extension classes. Note that this extension is not triangulable because $\mathbb{Q}$ is not a nilpotent matrix.

On the other hand, if we had taken $\mathfrak{u}_{n}$ instead of $\mathfrak{g l}_{n}$, then we would have $\mathbb{Q}=\beta(\mathbb{U})+\mathbb{U}^{2}$ where $\mathbb{U}$ is a strictly upper triangular matrix with $i j$-th entry equal to $x_{i j}$. It is clear that $\mathbb{Q}$ is also strictly upper triangular, so $\mathbb{Q}^{n}$ will be zero. In fact, $Q_{\mathfrak{u}_{n}}$ is a triangulable quadratic map.

Remark 6.7. The calculation we performed above in Example 6.6 can be used to see some of the earlier results in a different way. Let $E: 0 \rightarrow V \rightarrow G \rightarrow W \rightarrow 0$ be a Bockstein closed extension with binding operator $L$. Choosing a basis for $V$, we can view $L$ as a homomorphism $L: W \rightarrow \mathfrak{g l}_{n}\left(\mathbb{F}_{2}\right)$. Using $L$, we can lift the extension $E\left(Q_{\mathfrak{g l}_{n}}\right)$ and obtain

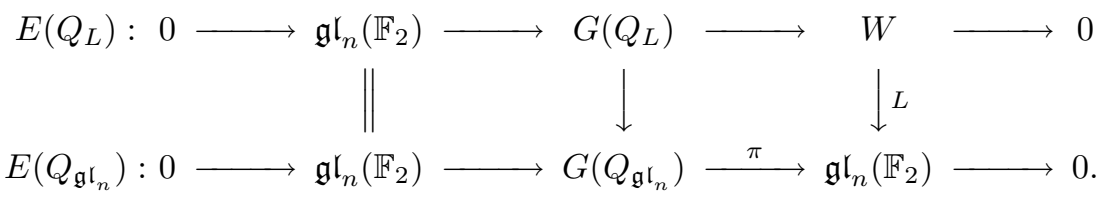

Since the extension class for the bottom extension is $\beta(\mathbb{X})+\mathbb{X}^{2}$, the extension class for $E\left(Q_{L}\right)$ will be $\beta(L)+L^{2}$. So, the class $\beta(L)+L^{2}$ can be thought of as the obstruction for lifting $L: W \rightarrow \mathfrak{g l}_{n}\left(\mathbb{F}_{2}\right)$ to a homomorphism $\widehat{L}: W \rightarrow G\left(Q_{\mathfrak{g l}_{n}}\right)$ such that $\pi \circ \widehat{L}=L$. Note that $L$ corresponds to a group homomorphism $\rho: W \rightarrow$ $K_{n}(\mathbb{Z} / 4)$ under the exp-log correspondence, and the map $\pi$ becomes the mod 4 reduction map

$$
K_{n}(\mathbb{Z} / 8) \rightarrow K_{n}(\mathbb{Z} / 4)
$$

when $K_{n}(\mathbb{Z} / 4)$ is identified with $\mathfrak{g l}_{n}\left(\mathbb{F}_{2}\right)$ (see Example 2.5). This shows that $\beta(L)+L^{2}$ is the obstruction for lifting the representation $\rho: W \rightarrow K_{n}(\mathbb{Z} / 4)$ to a representation of a $\mathbb{Z} / 8[W]$-lattice. This provides another way to see the equivalence $(i) \Leftrightarrow(i v)$ given in Proposition 5.5 . 


\section{Frattini And EFFECTIVE EXtensions}

In this section we consider Bockstein closed extensions with some additional conditions. These conditions are standard conditions one considers in group extension theory. We start with the following:

Definition 7.1. A central extension $E: 0 \rightarrow V \rightarrow G \rightarrow W \rightarrow 0$ is called a Frattini extension if the Frattini subgroup $\Phi(G)$ of $G$ is equal to $V$.

Recall that the Frattini subgroup of a $p$-group $G$ is defined as the subgroup $\Phi(G)=G^{p}[G, G]$ where $G^{p}$ is the subgroup generated by the $p$ th powers and $[G, G]$ is the commutator subgroup of $G$. In the case $p=2$, one has $\Phi(G)=G^{2}$, since in this case, $G / G^{2}$ has exponent 2 implies that it is abelian, so $[G, G] \leq G^{2}$. Because of this it makes sense to define the Frattini condition for quadratic maps as follows.

Definition 7.2. We say that a quadratic map $Q: W \rightarrow V$ is Frattini if the set $\Im(Q)=\{Q(w) \mid w \in W\}$ generates $V$. Equivalently, a quadratic map $Q$ is Frattini if the associated central extension $E(Q): 0 \rightarrow V \rightarrow G(Q) \rightarrow W \rightarrow 0$ is a Frattini extension.

Notice that when an extension of the form $E: 0 \rightarrow V \rightarrow G \rightarrow W \rightarrow 0$ is not Frattini, then $\Phi(G)$ is a proper subspace of $V$, and the group $G$ splits as $G^{\prime} \times \mathbb{Z} / 2$. In terms of group extension theory the trivial summand causes no extra difficulties, so to avoid trivialities, one often assumes that the extensions in question are Frattini extensions.

For Frattini extensions we have the following useful criteria:

Lemma 7.3. Let $E: 0 \rightarrow V \rightarrow G \rightarrow W \rightarrow 0$ be a Frattini extension with extension class $q=\left(q_{1}, \ldots, q_{n}\right)$ with respect to some basis for $V$. If $a_{1} q_{1}+\cdots+a_{n} q_{n}=0$ for some scalars $a_{1}, \ldots, a_{n}$, then $a_{1}=\cdots=a_{n}=0$.

Proof. If there exists $a_{1}, \ldots, a_{n} \in \mathbb{F}_{2}$ with $a_{1} q_{1}+\cdots+a_{n} q_{n}=0$, then one would have $a_{1} Q_{1}(w)+\cdots+a_{n} Q_{n}(w)=0$ for all $w \in W$. But, then $\Im(Q)$ will lie inside the kernel of the functional $a_{1} x_{1}+\cdots+a_{n} x_{n}$. The Frattini condition $\Im(Q)=V$ will hold only if this functional is zero.

We also have the following nice basis choice for Frattini extensions.

Lemma 7.4. Let $Q: W \rightarrow V$ be a Frattini extension where $W$ and $V$ are nonzero, and let $k$ be a positive integer such that $k \leq \min \{\operatorname{dim} V, \operatorname{dim} W\}$. Then, there exists a set of linearly independent vectors $\left\{w_{1}, \ldots, w_{k}\right\}$ in $W$ such that $\left\{Q\left(w_{1}\right), \ldots, Q\left(w_{k}\right)\right\}$ is a linearly independent set in $V$.

Proof. Let $m=\min \{\operatorname{dim} V, \operatorname{dim} W\}$. For $k=1$, the lemma is clear, since there is always a non-zero vector $w$ in $W$ whose image is non-zero; otherwise $V$ will be the zero space. Assume that the lemma is true for some $k<m$. We will show that it also holds for $k+1$.

By assumption there is a linearly independent set $\left\{w_{1}, \ldots, w_{k}\right\}$ of vectors in $W$ such that $\left\{Q\left(w_{1}\right), \ldots, Q\left(w_{k}\right)\right\}$ is a linearly independent set in $V$. Let $W_{k}$ be the subspace of $W$ generated by $\left\{w_{1}, \ldots, w_{k}\right\}$, and $V_{k}$ the subspace of $V$ generated by $\left\{Q\left(w_{1}\right), \ldots, Q\left(w_{k}\right)\right\}$. Then, $\operatorname{dim} W_{k}=\operatorname{dim} V_{k}=k<m$. To complete the proof we need to show that there exists a $w$ in the set difference $W-W_{k}$ such that $Q(w) \notin V_{k}$. Assume to the contrary that there is no such $w \in W$, i.e., $Q(w)$ lies in 
$V_{k}$ for all $w \in W-W_{k}$. Now fix a $w \in W-W_{k}$. Note that for all $i=1, \ldots, k$, we have $Q\left(w+w_{i}\right) \in V_{k}$. Therefore, for all $1 \leq i \leq k$, we have

$$
B\left(w, w_{i}\right)=Q\left(w+w_{i}\right)+Q(w)+Q\left(w_{i}\right) \in V_{k} .
$$

By bilinearity, we get $B\left(w, w^{\prime}\right) \in V_{k}$ for all $w^{\prime} \in W_{k}$. Since this statement is true for all $w \in W-W_{k}$, we have

$$
B\left(W-W_{k}, W_{k}\right) \subseteq V_{k} .
$$

Now, take $w \in W-W_{k}$ and $w^{\prime} \in W_{k}$. Since $B\left(w, w^{\prime}\right), Q(w)$, and $Q\left(w+w^{\prime}\right)$ are all in $V_{k}$, we can conclude that

$$
Q\left(w^{\prime}\right)=B\left(w, w^{\prime}\right)+Q(w)+Q\left(w+w^{\prime}\right)
$$

is in $V_{k}$. Thus $Q\left(w^{\prime}\right) \in V_{k}$ for every $w^{\prime} \in W_{k}$. For vectors in $W-W_{k}$ we assumed at the beginning that their image under $Q$ lies in $V_{k}$, so we obtained that $Q(w)$ lies in $V_{k}$ for all $w \in W$. But $V_{k}$ is a proper subspace of $V$ since $\operatorname{dim} V_{k}=k<m \leq \operatorname{dim} V$. This contradicts the assumption that $Q$ is Frattini.

This last lemma, in particular, tells us that if $\operatorname{dim} V=\operatorname{dim} W=n$, then there exists a basis $\left\{w_{1}, \ldots, w_{n}\right\}$ for $W$ and a basis $\left\{v_{1}, \ldots, v_{n}\right\}$ for $V$ such that $Q\left(w_{i}\right)=$ $v_{i}$ for all $i=1, \ldots, n$. In this case, the extension class $q=\left(q_{1}, \ldots, q_{n}\right)$ is in the form

$$
q_{k}=x_{k}^{2}+\sum_{i<j} \gamma_{i j}^{(k)} x_{i} x_{j}
$$

for all $k=1, \ldots, n$. If the extension class is written in this form, we say it is in bijective form, and the basis which is induced from this form is called a bijective basis.

Note that in the case where $\operatorname{dim} W=\operatorname{dim} V=n$, a bijective basis also allows us to identify $W$ with $V$ and write quadratic maps as operators on one vector space. We will use this later in the paper.

Now, we will impose another property for the extensions $E(Q)$.

Definition 7.5. We say that a quadratic map $Q: W \rightarrow V$ is effective if

$$
\operatorname{Ker}(Q)=\{w \in W \mid Q(w)=0\}=\{0\} .
$$

An extension $E(Q): 0 \rightarrow V \rightarrow G(Q) \rightarrow W \rightarrow 0$ is called effective if the associated quadratic map $Q$ is effective.

Equivalent interpretations of effective extensions are given in the following proposition:

Proposition 7.6. Let $E(Q): 1 \rightarrow V \rightarrow G(Q) \rightarrow W \rightarrow 1$ be a central extension with quadratic map $Q$ and extension class $q$. Then the following are equivalent:

(i) $E(Q)$ is effective, i.e., $Q(w)=0$ implies $w=0$ for all $w \in W$.

(ii) $\operatorname{Res}_{\langle w\rangle}^{W}(q) \neq 0$ for any nonzero cyclic subgroup $\langle w\rangle$ of $W$.

(iii) $V$ is a maximal elementary abelian subgroup of $G(Q)$.

(iv) All the elements of order 2 in $G(Q)$ lie in $V$.

Proof. It is easy to see that $(i) \Leftrightarrow(i i)$ by using the fact that any factor set $f$ representing $q$ has $f(w, w)=Q(w)$. Note that on a one dimensional subspace $\langle w\rangle$ of $W$, the vector $Q(w)=f(w, w)$ determines whether $Q$ and $q$ are zero when restricted to that subspace. The implication $(i) \Rightarrow($ iii $)$ is clear since if $V$ is not maximal, then one can find a nontrivial element $w \in W$ coming from the larger 
elementary abelian subgroup such that $Q(w)=(\hat{w})^{2}=0$. On the other hand if we have (iii) and $g \in G$ has order 2 , since $V$ is central in $G$, we have that $\langle g, V\rangle$ is an elementary abelian 2-group. Since $V$ is maximal, it must be that $g \in V$. Thus $(i i i) \Rightarrow(i v)$. Finally, $(i v) \Rightarrow(i)$ since if there is a lift $\hat{w} \in G-V$ such that $(\hat{w})^{2}=1$, then there exists a nonzero $w \in W$ such that $Q(w)=0$.

Note that since a nontrivial zero of $Q: W \rightarrow V$ corresponds to a nontrivial common zero of the homogeneous quadratic polynomials $Q_{1}, \ldots, Q_{m}: W \rightarrow \mathbb{F}_{2}$, the Chevalley-Warning theorem tells us that if $E$ is an effective extension, then $\operatorname{dim} W \leq 2 \operatorname{dim} V$. This bound can be seen to be strict by considering the effective central extension for $G=\left(Q_{8}\right)^{m}$, where $Q_{8}$ is the quaternionic group of order 8 given by

$$
0 \rightarrow(\mathbb{Z} / 2)^{m} \rightarrow\left(Q_{8}\right)^{m} \rightarrow(\mathbb{Z} / 2)^{2 m} \rightarrow 0,
$$

where $\operatorname{dim} W=2 m$ and $\operatorname{dim} V=m$.

For $Q: \mathfrak{g l}_{n}\left(\mathbb{F}_{2}\right) \rightarrow \mathfrak{g l}_{n}\left(\mathbb{F}_{2}\right)$, one has $Q(\mathbb{A})=\mathbb{O}$ if and only if $\mathbb{A}^{2}=\mathbb{A}$ (i.e., $\mathbb{A}$ is a projection). Thus if $W$ is a square-closed subspace, $Q: W \rightarrow W$ is effective whenever $W$ does not contain any nonzero projections. Thus for example $W=$ $\mathfrak{u}_{n}$, the strictly upper triangular matrices (since the only projection with all zero eigenvalues is the zero projection).

Definition 7.7. Let $f_{1}, \ldots, f_{m}$ be a sequence of polynomials in $\mathbb{F}_{2}\left[x_{1}, \ldots, x_{n}\right]$. The sequence $f_{1}, \ldots, f_{m}$ is called a regular sequence if $f_{k}$ is a non-zero divisor in the quotient ring $\mathbb{F}_{2}\left[x_{1}, \ldots, x_{n}\right] /\left(f_{1}, \ldots, f_{k-1}\right)$ for all $k=1, \ldots, n$.

We have the following important fact about extensions which are both Bockstein closed and effective:

Proposition 7.8 (Serre, Quillen, Carlsson). Let E be a Bockstein closed, effective extension. Then, $\operatorname{dim} W \leq \operatorname{dim} V$. Moreover, if $\operatorname{dim} V=\operatorname{dim} W$, then the components $q_{1}, \ldots, q_{n}$ of the extension class (and any permutation of them) form a regular sequence.

Proof. Let $k$ denote the algebraic closure of $\mathbb{F}_{2}$. When $E$ is Bockstein closed, the associated ideal $I(Q)$ generated by the components of the quadratic map is closed under the Steenrod operations. Then, by a result of Serre [7], the variety of $I(Q)$ over $k$ must include a non-trivial rational point if it is a nonzero variety. (Note since $I(Q)$ is a homogeneous ideal, and zero will always be in its corresponding variety. Serre's theorem says that if the variety has some other nontrivial solution over $k$, it will also have a nontrivial solution over $\mathbb{F}_{2}$.) By the effectiveness condition, the components $q_{1}, \ldots, q_{n}$ of the extension class $q$ have no nontrivial common zero in $W$. So, $q_{1}, \ldots, q_{n}$ have no nontrivial common zero in $k$, by Serre's theorem. So, $n \leq m$ where $m=\operatorname{dim} W$ and $n=\operatorname{dim} V$ as before.

For the second part we use a result in commutative algebra which states that a sequence of polynomials $f_{1}, \ldots, f_{n}$ in $m$ variables is a regular sequence if

$$
\operatorname{dim} \operatorname{Var}\left(f_{1}, \ldots, f_{m}\right) \leq m-n
$$

(see, for example, [5]). Since $m-n=0=\operatorname{dim} \operatorname{Var}\left(q_{1}, \ldots, q_{n}\right)$, we can conclude that $q_{1}, \ldots, q_{n}$ (or any permutation of them) is a regular sequence. 
Thus we are motivated to make the following definition:

Definition 7.9. A central extension

$$
E(Q): 0 \rightarrow V \rightarrow G(Q) \rightarrow W \rightarrow 0
$$

with corresponding quadratic map $Q: W \rightarrow V$ is called 2-power exact if the following 3 conditions hold:

(a) $\operatorname{dim}(V)=\operatorname{dim}(W)$.

(b) The extension is Frattini, i.e., $\Im(Q)=V$.

(c) The extension is effective, i.e., $Q(w)=0$ if and only if $w=0$.

In the next section we will study Bockstein closed 2-power exact extensions. Note that if $E$ is a Bockstein closed extension satisfying only the conditions $(a)$ and $(c)$ above, then we can conclude that it is 2-power exact. To see this, observe that the quadratic map $Q: W \rightarrow \Im(Q)$ is Bockstein closed and effective, so by Proposition 7.8, we should have $\operatorname{dim} W \leq \operatorname{dim} \Im(Q)$. By condition $(a)$, we have $\operatorname{dim} W=\operatorname{dim} V$, so we can conclude $\Im(Q)=V$.

Note that a typical example of a 2-power exact extension is the extension associated with the $\mathfrak{g l}$-induced quadratic map $Q: \mathfrak{u}_{n}\left(\mathbb{F}_{2}\right) \rightarrow \mathfrak{u}_{n}\left(\mathbb{F}_{2}\right)$, where $\mathfrak{u}_{n}\left(\mathbb{F}_{2}\right)$ is the $\mathbb{F}_{2}$-vector space of strictly upper triangular matrices.

\section{Bockstein CLOSED 2-POWER EXACT SEQUENCES}

In this section we consider Bockstein closed 2-power exact extensions and prove some restrictions on binding operators of these extensions. This leads to an interesting group theoretical result for such extensions. We start with the following:

Proposition 8.1. Let $E(Q): 0 \rightarrow V \rightarrow G(Q) \rightarrow W \rightarrow 0$ be a Bockstein closed 2-power exact sequence corresponding to the quadratic map $Q: W \rightarrow V$. Then, there is a unique binding operator for $Q$.

Proof. Let $L_{1}, L_{2} \in H^{1}(W, \operatorname{End}(V))$ such that $\beta(q)=L_{1} q=L_{2} q$. Then we have $\left(L_{1}+L_{2}\right) q=0$. Choosing a basis for $W$ and $V$, we can express $q$ as a column vector and $L$ as an $n \times n$ matrix with entries in $H^{1}\left(W, \mathbb{F}_{2}\right)$. So, equation $\left(L_{1}+L_{2}\right) q=0$ gives a system of equations in $q_{i}$ 's with coefficients in $H^{1}\left(W, \mathbb{F}_{2}\right)$. By Proposition 7.8, the entries of $L_{1}+L_{2}$ must lie in $I(Q)$. Since the entries are one dimensional and $I(Q)$ is generated by 2 -dimensional classes, we have $L_{1}+L_{2}=0$.

Another consequence of regularity is the following:

Proposition 8.2. Let $E(Q): 0 \rightarrow V \rightarrow G(Q) \rightarrow W \rightarrow 0$ be a Bockstein closed 2 -power exact sequence corresponding to the quadratic map $Q: W \rightarrow V$. Choose a bijective basis for $V$ and $W$ as in Lemma 7.4, and let $L=\sum_{i} L_{i} x_{i}$ be the binding matrix with respect to this basis. Then, we have

$$
\beta(L)+L^{2}=\sum_{i}\left(L_{i}^{2}+L_{i}\right) q_{i}
$$

Proof. Applying the Bockstein operator on $\beta(q)=L q$, we get $\left[\beta(L)+L^{2}\right] q=0$. Since $q_{1}, \ldots, q_{n}$ is a regular sequence (in any order), each entry of $\beta(L)+L^{2}$ must be in the ideal $I(q)$. So, we have

$$
\beta(L)+L^{2}=\sum_{i} K_{i} q_{i}
$$


for some $n \times n$-matrices $K_{1}, K_{2}, \ldots, K_{n}$. Note that

$$
\beta(L)+L^{2}=\sum_{i}\left(L_{i}^{2}+L_{i}\right) x_{i}^{2}+\sum_{i<j}\left[L_{i}, L_{j}\right] x_{i} x_{j} .
$$

Since $q=\left(q_{1}, \ldots, q_{n}\right)$ is in the bijective form, i.e.,

$$
q_{i}=x_{i}^{2}+\sum_{l<k} \gamma_{l k}^{(i)} x_{l} x_{k}
$$

for each $i$, we have

$$
\sum_{i} K_{i} q_{i}=\sum_{i} K_{i} x_{i}^{2}+\sum_{i} \sum_{l<k} K_{i} \gamma_{l k}^{(i)} x_{l} x_{k}
$$

Comparing the coefficients of $x_{i}^{2}$, we get $K_{i}=L_{i}^{2}+L_{i}$ for each $i=1, \ldots, n$. This completes the proof.

An immediate consequence of Proposition 8.2 is the following:

Proposition 8.3. Let $E(Q): 0 \rightarrow V \rightarrow G(Q) \rightarrow W \rightarrow 0$ be a Bockstein closed 2 -power exact sequence corresponding to the quadratic map $Q: W \rightarrow V$. Suppose that we have chosen a bijective basis for $V$ and $W$ as in Lemma 7.4 , and let $L=$ $\sum_{i} L_{i} x_{i}$ be the binding matrix with respect to this basis. Then, the following diagram commutes:

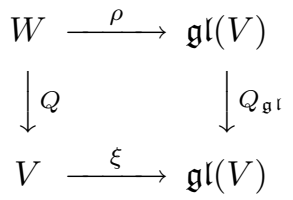

where $\rho$ and $\xi$ are linear transformations such that $\rho\left(w_{i}\right)=L_{i}$ and $\xi\left(v_{i}\right)=L_{i}^{2}+L_{i}$ for all $i=1, \ldots, n$.

Proof. We will show that the quadratic maps $\xi \circ Q$ and $Q_{\mathfrak{g r}} \circ \rho$ are equal by showing that the corresponding cohomology classes $\xi_{*}(q)$ and $\rho^{*}\left(q_{\mathfrak{g l}}\right)$ are equal, where

$$
H^{2}(W, V) \stackrel{\xi_{*}}{\longrightarrow} H^{2}(W, \mathfrak{g l}(V)) \stackrel{\rho^{*}}{\longleftarrow} H^{2}(\mathfrak{g l}(V), \mathfrak{g l}(V)) .
$$

Note that $\xi_{*}(q)=\sum_{i}\left(L_{i}^{2}+L_{i}\right) q_{i}$. On the other hand $\rho^{*}\left(q_{\mathfrak{g l}}\right)$ is equal to $\rho^{*}(\beta(\mathbb{X})+$ $\left.\mathbb{X}^{2}\right)=\beta(L)+L^{2}$ (see Example 6.6). By Proposition 8.2 these two are equal.

The commuting diagram given in Proposition 8.3 can be thought of as a representation of a quadratic map. In terms of group extensions we have:

Corollary 8.4. The following diagram commutes:

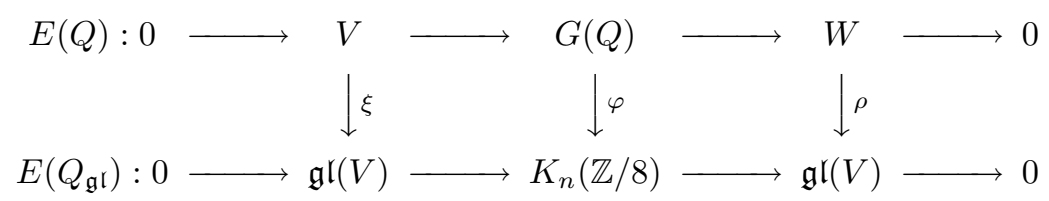

where $\xi$ and $\rho$ are as above.

We will usually refer to such a diagram as a morphism of group extensions. We can think of this morphism as a representation of $E(Q)$ into a $\mathfrak{g l}$-induced extension. Whenever there is such a morphism, we can define its kernel as the extension

$$
0 \rightarrow \operatorname{ker} \xi \rightarrow \operatorname{ker} \varphi \rightarrow \operatorname{ker} \rho \rightarrow 0
$$


whose associated quadratic map is given by $\left.Q\right|_{\text {ker } \rho}: \operatorname{ker} \rho \rightarrow \operatorname{ker} \xi$. Note that the representation given by the binding operator in general has a non-trivial kernel. When this happens one can express the original extension as an extension of group extensions with smaller rank. The following is an example of this situation:

Example 8.5. Let $E(Q)$ be the extension with extension class

$$
q=\left(x^{2}+y z, y^{2}+x z, z^{2}\right)
$$

This extension was considered earlier in Example 5.4, and using the calculations there, it is easy to see that the images of both $\rho$ and $\xi$ have rank 2 , so the image of $\varphi$ is an extension of the form

$$
E\left(Q^{\prime}\right): 0 \rightarrow(\mathbb{Z} / 2)^{2} \rightarrow \operatorname{im} \varphi \rightarrow(\mathbb{Z} / 2)^{2} \rightarrow 0 .
$$

Further calculations show that the extension class for $E\left(Q^{\prime}\right)$ is $q^{\prime}=\left(s^{2}+s t, t^{2}\right)$. In this case, the kernel is the extension

$$
E\left(Q^{\prime \prime}\right): 0 \rightarrow \mathbb{Z} / 2 \rightarrow \operatorname{ker} \varphi \rightarrow \mathbb{Z} / 2 \rightarrow 0
$$

where $q^{\prime \prime}=u^{2}$.

Remark 8.6. Note that the situation given in Example 8.5 can be best described by saying that we have a "central extension" of group extensions of the form

$$
0 \rightarrow E\left(Q^{\prime \prime}\right) \rightarrow E(Q) \rightarrow E\left(Q^{\prime}\right) \rightarrow 0
$$

It is possible to develop an extension theory for group extensions (or for quadratic maps) together with an appropriate representation theory and make sense of this. We leave this to another paper which we plan to write as a sequel to this paper.

Another observation we can make about Example 8.5 is that the quadratic map $q$ is triangulable since both $q^{\prime \prime}$ and $q^{\prime}$ are triangulable. In fact, the triangulation will come from a basis $\left\{v_{1}, v_{2}, v_{3}\right\}$ such that $v_{1}$ generates the kernel of $\xi$. One may ask if in general the morphism of extensions obtained from the binding operator gives us a triangulation (possibly by applying the procedure repeatedly).

We should also mention here that we do not have any examples of non-triangulable Bockstein closed 2-power exact extensions. There is not enough evidence to claim that every Bockstein closed 2-power exact extension is triangulable, but it is certainly tempting to ask if it is true. The following shows that to find a nontriangulable Bockstein closed extension counterexample one needs at least $\operatorname{dim} V \geq$ 3.

Proposition 8.7. Let $E: 0 \rightarrow V \rightarrow G \rightarrow W \rightarrow 0$ be a Bockstein closed extension. If $\operatorname{dim} V=2$, then $E$ is diagonalizable.

Proof. Let $q=\left(q_{1}, q_{2}\right)$ be the extension class for $E$. We can assume the extension is Frattini, because otherwise the result holds for trivial reasons. Since $E$ is Bockstein closed, there is an

$$
L=\left[\begin{array}{ll}
f & g \\
h & k
\end{array}\right] \text { such that } \quad\left[\begin{array}{l}
\beta\left(q_{1}\right) \\
\beta\left(q_{2}\right)
\end{array}\right]=\left[\begin{array}{ll}
f & g \\
h & k
\end{array}\right]\left[\begin{array}{l}
q_{1} \\
q_{2}
\end{array}\right] .
$$

Applying the Bockstein operator again, we get

$$
\left[\beta(L)+L^{2}\right] q=\left[\begin{array}{cc}
g h & g(g+f+k) \\
h(h+f+k) & g h
\end{array}\right]\left[\begin{array}{l}
q_{1} \\
q_{2}
\end{array}\right]=0
$$


If $g=0$, then the above equation gives $h(h+f+k) q_{1}=0$, which implies $h(h+f+k)$ by the Frattini condition. So, $\beta(L)+L^{2}=0$, and hence $L$ is diagonalizable. Similarly, one can show that $L$ is diagonalizable when $h=0$ as well. So, assume that both $g$ and $h$ are non-zero. Then, we get

$$
\left[\begin{array}{cc}
h & g+f+k \\
h+f+k & g
\end{array}\right]\left[\begin{array}{l}
q_{1} \\
q_{2}
\end{array}\right]=0
$$

This gives $(f+k)\left(q_{1}+q_{2}\right)=0$, so we get $f=k$ by the Frattini condition. Setting $f+k=0$ in the above matrix equation, we get $h q_{1}+g q_{2}=0$. Using this we get

$$
\left[\begin{array}{l}
\beta\left(q_{1}\right) \\
\beta\left(q_{2}\right)
\end{array}\right]=\left[\begin{array}{cc}
f+h & 0 \\
0 & g+k
\end{array}\right]\left[\begin{array}{l}
q_{1} \\
q_{2}
\end{array}\right]
$$

Hence, $E$ is diagonalizable.

\section{Strongly Bockstein Closed exact sequences}

The main examples of Bockstein closed quadratic maps are $\mathfrak{g l}$-induced extensions

$$
E(Q): 0 \rightarrow W \rightarrow G(Q) \rightarrow W \rightarrow 0,
$$

where $W$ is a square closed subspace of $\mathfrak{g l}_{n}\left(\mathbb{F}_{2}\right)$ and the quadratic map $Q: W \rightarrow W$ is given by $Q(\mathbb{A})=\mathbb{A}^{2}+\mathbb{A}$. In this case the associated bilinear map $B(\mathbb{A}, \mathbb{B})=$ $\mathbb{A} \mathbb{B}+\mathbb{B} \mathbb{A}$ satisfies the equation

$$
B(Q(\mathbb{A}), \mathbb{B})=B(\mathbb{A}, \mathbb{B})+B(B(\mathbb{A}, \mathbb{B}), \mathbb{A}),
$$

so the equation in Theorem 3.6 holds with $P=B$. These extensions are Bockstein closed in a stronger sense.

Definition 9.1. Let $Q: W \rightarrow W$ be a quadratic map and $B: W \times W \rightarrow W$ be the associated bilinear map. We say $Q$ is strongly Bockstein closed if the equation

$$
B(Q(x), y)=B(x, y)+B(B(x, y), x)
$$

holds for all $x, y \in W$. Equivalently, a quadratic map is strongly Bockstein closed if the equation $\beta(q)=L q$ holds with $L=A d_{B}$, where $A d_{B}: W \rightarrow \operatorname{End}(W)$ is the adjoint operator defined as $A d_{B}(x)(y)=B(x, y)$.

There is a close connection between strongly Bockstein closed extensions and 2-restricted Lie algebras. To explain this connection, we first recall the definition of 2-restricted Lie algebras.

Definition 9.2. Let $W$ be a vector space over $\mathbb{F}_{2}$. We say that $\mathfrak{g}=\left(W,[\cdot, \cdot],(\cdot)^{[2]}\right)$ is a 2-restricted Lie algebra if the following hold for all $x, y \in W$ :

(i) $(x+y)^{[2]}=x^{[2]}+y^{[2]}+[x, y]$,

(ii) $\left[x^{[2]}, y\right]=[[x, y], x]$.

Let $Q: W \rightarrow W$ be a strongly Bockstein closed quadratic map, and let $B$ : $W \times W \rightarrow W$ be the bilinear map associated to $Q$. If we take $B$ as a bracket and define the 2-power map on $W$ by the formula $w^{[2]}=Q(w)+w$, then $W$ becomes a restricted Lie algebra together with this bracket and 2-power map. Conversely, if 
$\mathfrak{g}=\left(W,[\cdot, \cdot],(\cdot)^{[2]}\right)$ is a 2-restricted Lie algebra, then the quadratic map defined by $Q(w)=w^{[2]}+w$ is a strongly Bockstein closed quadratic map. We conclude the following:

Proposition 9.3. There is a one-to-one correspondence between strongly Bockstein closed extensions and 2-restricted Lie algebras $\mathfrak{g}=\left(W,[\cdot, \cdot],(\cdot)^{[2]}\right)$. The correspondence is given by the formulas $Q(w)=w^{[2]}+w$ and $B\left(w, w^{\prime}\right)=\left[w, w^{\prime}\right]$ where $B$ is the bilinear map associated to $Q$.

Recall that a representation of a 2-restricted Lie algebra is a linear transformation

$$
\rho: W \rightarrow \mathfrak{g l}(V)
$$

which commutes with brackets and 2-power maps, where the 2-power map on $\mathfrak{g l}(V)$ is given by $A \rightarrow A^{2}$. Note that given a representation $\rho: W \rightarrow \mathfrak{g l}(V)$ of 2-restricted Lie algebras, we have a commuting diagram of the form

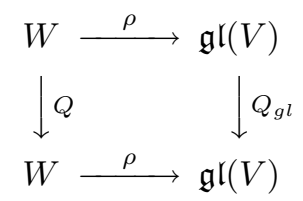

where $Q$ is the associated quadratic map to the 2-power map on $W$, and $Q_{\mathfrak{g l}}$ is the quadratic map $A \rightarrow A^{2}+A$ as before.

Since every 2-restricted Lie algebra $\mathfrak{g}$ has a faithful restricted Lie algebra representation $\rho: W \rightarrow \mathfrak{g l}_{k}\left(\mathbb{F}_{2}\right)$ for some $k$ (see, for example, page 192 of [3]), every strongly Bockstein closed quadratic map has a commuting diagram of the above form with injective horizontal maps. From this, we conclude the following:

Proposition 9.4. If $Q: W \rightarrow W$ is a strongly Bockstein closed quadratic map, then it is a $\mathfrak{g l}$-induced quadratic map.

In the rest of the section we consider strongly Bockstein closed extensions which are 2-power exact. It turns out that these are the true analogues of Bockstein closed $p$-power exact extensions with $p>2$. We now explain this analogy.

Let $E: 0 \rightarrow V \rightarrow G \rightarrow W \rightarrow 0$ be a $p$-power exact extension where $V$ and $W$ are $\mathbb{F}_{p}$-vector spaces with $p>2$. Recall that $E$ is $p$-power exact means that $\operatorname{dim} W=\operatorname{dim} V$ and that $E$ is both Frattini and effective. The cohomology of these extensions has been studied by Browder and Pakianathan in [1]. One of the main ingredients for their analysis is the fact that the $p$-power map gives an isomorphism $W \rightarrow V$ under the assumption of $p$-power exactness. Using the inverse of the $p$ power map, they turned the bilinear commutator map $[\cdot, \cdot]: W \times W \rightarrow V$ into a bracket $[\cdot, \cdot]: W \times W \rightarrow W$. Then they showed that the extension is Bockstein closed if and only if this bracket is a Lie bracket.

For $p=2$, the general situation is much more complicated, but the case of strongly Bockstein closed extensions is, in fact, very similar to the $p>2$ case. As before let

$$
E(Q): 0 \rightarrow V \rightarrow G(Q) \rightarrow W \rightarrow 0
$$

denote a 2-power exact sequence corresponding to the quadratic map $Q: W \rightarrow$ $V$. By Lemma 7.4, we can choose bijective bases $\left\{w_{1}, \ldots, w_{n}\right\}$ and $\left\{v_{1}, \ldots, v_{n}\right\}$ such that $Q\left(w_{i}\right)=v_{i}$ for all $i=1, \ldots, n$. Let $\left\{x_{1}, \ldots, x_{n}\right\}$ be the basis dual to 
$\left\{w_{1}, \ldots, w_{n}\right\}$, and let $\phi: V \rightarrow W$ be the linear map which takes $v_{i}$ to $w_{i}$ for all $i=1, \ldots, n$. We can replace $Q$ with a quadratic map $\phi \circ Q: W \rightarrow W$ and bilinear map $B$ with $\phi \circ B: W \times W \rightarrow W$. We will still denote them by $Q$ and $B$ as before. With respect to the basis $\left\{w_{1}, \ldots, w_{n}\right\}$, we have the following matrix representation:

$$
q=\beta(x)+A x,
$$

where $x$ is the column matrix with $i$-th entry equal to $x_{i}$, and $A$ is a matrix whose entries are linear polynomials in the $x_{i}$ 's. In fact, we can describe $A$ in terms of a bilinear form $B$ as follows:

Let $A=\sum_{i=1}^{n} A_{i} x_{i}$. Then $A_{i}(j, k)=B_{j}\left(w_{i}, w_{k}\right)$ if $i<k$ and zero otherwise. Similarly, we can define $A^{\perp}$ as the matrix $A^{\perp}=\sum_{i=1}^{n} A_{i}^{\perp} x_{i}$, where $A_{i}^{\perp}(j, k)=$ $B_{j}\left(w_{i}, w_{k}\right)$ if $i>k$ and zero otherwise. Note that $A+A^{\perp}$ is the matrix for the adjoint map $A d_{B}: W \rightarrow \operatorname{Hom}(W, W)$ defined by $A d_{B}(w)\left(w^{\prime}\right)=B\left(w, w^{\prime}\right)$.

Applying Bockstein to Equation (6), we get

$$
\beta(q)=\beta(A) x+A \beta(x)=\beta(A) x+A^{2} x+A q .
$$

We can write $\beta(A) x=A^{\perp} \beta(x)=A^{\perp} q+A^{\perp} A x$. So we have

$$
\beta(q)=\left(A d_{B}\right) A x+\left(A d_{B}\right) q .
$$

For $p>2$, the above equation holds only when $\left(A d_{B}\right) A x=0$ and $\beta(q)=\left(A d_{B}\right) q$. This has two consequences: One is that for $p>2$ an extension is Bockstein closed only if the $A d_{B}$ is a binding operator, i.e., Bockstein closedness is the same as strongly Bockstein closedness. The second consequence is that an extension is Bockstein closed only if $A d_{B}$ satisfies a specific equation $\left(A d_{B}\right) A x=0$. So it is much easier to decide on Bockstein closedness for $p>2$. Moreover, it turns out that in this case the equation $\left(A d_{B}\right) A x=0$ is equivalent to the fact that the bilinear map $B: W \times W \rightarrow W$ is a Lie bracket, giving the result of Browder and Pakianathan [1] that the extension is Bockstein closed if and only if the associated bracket $B$ is a Lie bracket.

In the case $p=2$, we do not have such a strong conclusion. In general, one can have Bockstein closed extensions without the equation $\left(A d_{B}\right) A x=0$ holding. However, in the case of strongly Bockstein closed extensions, one has $\beta(q)=A d_{B} q$, forcing $\left(A d_{B}\right) A x=0$ to hold. For $p=2$, the equation $\left(A d_{B}\right) A x=0$ is equivalent to the condition that $B$ is a Lie bracket for a 2-restricted Lie algebra with 2-power map given by $w^{[2]}=Q(w)+w$. So, as in the case of $p>2$, we have a connection with Lie algebras, but instead of with the usual Lie algebras, with restricted Lie algebras. Thus:

Lemma 9.5. There is a 1-1 correspondence between strongly Bockstein closed 2-power exact quadratic maps $Q: W \rightarrow W$, and 2-restricted Lie algebras $\mathfrak{g}=$ $\left(W,[\cdot, \cdot],(\cdot)^{[2]}\right)$ satisfying the following properties:

(i) the elements of the form $w^{[2]}+w$ generate $W$,

(ii) for all $w \in W, w^{[2]}=w$ implies $w=0$.

The correspondence is given by the equation $Q(w)=w^{[2]}+w$.

If a 2-restricted Lie algebra satisfies the properties listed in the above lemma, we call this Lie algebra a 2-power exact restricted Lie algebra. It is easy to give an example of such Lie algebras. 
Example 9.6. We have seen earlier that the vector space of $n \times n$ strictly upper triangular matrices, denoted by $\mathfrak{u}_{n}$, forms a 2-restricted Lie algebra such that the quadratic map defined by $Q(\mathbb{A})=\mathbb{A}^{2}+\mathbb{A}$ is effective and Frattini. So, $\mathfrak{u}_{n}$ is a 2-power exact restricted Lie algebra.

We would like to remark that 2-power exact restricted Lie algebras are quite special. In particular we have the following result:

Proposition 9.7. Let $\mathfrak{g}$ be a 2-power exact restricted Lie algebra. If $\mathfrak{g}$ has a filtration with ideals

$$
0=\mathfrak{g}_{0} \subseteq \mathfrak{g}_{1} \subseteq \cdots \subseteq \mathfrak{g}_{k}=\mathfrak{g}
$$

such that $\operatorname{dim}\left(\mathfrak{g}_{i} / \mathfrak{g}_{i-1}\right)=1$, then $\mathfrak{g}$ is nilpotent.

Proof. Let $\mathfrak{g}_{1}=\left\langle x_{0}\right\rangle$. There is a linear map $\alpha: \mathfrak{g} \rightarrow \mathbb{F}_{2}$ such that $\left[x_{0}, x\right]=\alpha(x) x_{0}$ for every $x \in \mathfrak{g}$. If $x=w^{[2]}+w$ for some $w$, then

$$
\left[x_{0}, x\right]=\left[x_{0}, w^{[2]}+w\right]=\left[\left[x_{0}, w\right], w\right]+\left[x_{0}, w\right]=\left[\alpha(w)^{2}+\alpha(w)\right] x_{0}=0 .
$$

Since the elements of the form $w^{[2]}+w$ generate $W$, we can conclude that $x_{0}$ is central. So, the result follows from the following lemma.

Lemma 9.8. Let $\mathfrak{g}$ be a 2-power exact restricted Lie algebra, and let $Z(\mathfrak{g})$ be the center of $\mathfrak{g}$. Then, $\mathfrak{g} / Z(\mathfrak{g})$ is also a 2-power exact restricted Lie algebra with 2-power map defined by $x+Z(\mathfrak{g}) \rightarrow x^{[2]}+Z(\mathfrak{g})$.

Proof. It is easy to see that $\mathfrak{g} / Z(\mathfrak{g})$ satisfies the Frattini condition. Now we will show that it is effective. Assume $x+x^{[2]}=z$ for some $z \in Z(\mathfrak{g})$. Since the map defined by $x \rightarrow x^{[2]}+x$ is linear on $Z(\mathfrak{g})$, it must be an isomorphism by the effectiveness condition. So, we can find a $y \in Z(\mathfrak{g})$ such that $y^{[2]}+y=z$. Then, we have

$$
(x+y)^{[2]}=x^{[2]}+y^{[2]}=(x+z)+(y+z)=x+y .
$$

By the effectiveness of $\mathfrak{g}$, we must have $x+y=0$. So, $x \in Z(\mathfrak{g})$ giving that $\mathfrak{g} / Z(\mathfrak{g})$ is 2-power exact.

We do not know if all the 2-power exact restricted Lie algebras are nilpotent. It would be interesting to see whether the general theory of restricted Lie algebras applies to answer this question. We leave this as an open problem.

\section{ACKNowledgments}

We thank the referee for his comments on the paper.

\section{REFERENCES}

[1] W. Browder and J. Pakianathan, Cohomology of uniformly powerful p-groups, Trans. Amer. Math. Soc. 352 (2000), 2659-2688. MR1661313 (2000j:20099)

[2] K. Brown, Cohomology of Groups, Graduate Texts in Mathematics 87, Springer-Verlag, New York, 1994. MR 1324339 (96a:20072)

[3] N. Jacobson, Lie Algebras, Dover, New York, 1962. MR0143793 (26:1345)

[4] P. A. Minh and P. Symonds, The cohomology of pro-p groups with a powerfully embedded subgroup, J. Pure Appl. Algebra, 189 (2004), 221-246. MR2038572 (2004k:20113)

[5] D. Quillen, The Mod 2 Cohomology Rings of Extra-Special 2-Groups and the Spinor Groups, Math. Ann. 194 (1971), 197-212. MR0290401 (44:7582) 
[6] D. Rusin, The 2-groups of rank 2, J. Algebra 149 (1992), 1-31. MR1165197 (93d:20042)

[7] J. P. Serre, Sur la Dimension Cohomologique des Groupes Profinis, Topology 3 (1965), 413420. MR 0180619 (31:4853)

Department of Mathematics, University of Rochester, Rochester, New York 14627

E-mail address: jonpak@math.rochester.edu

Department of Mathematics, Bilkent University, Ankara, 06800, Turkey

E-mail address: yalcine@fen.bilkent.edu.tr 